\title{
An ancestral interaction module promotes oligomerization in divergent mitochondrial ATP synthases
}

\section{Alexey Amunts ( $\square$ amunts@scilifelab.se )}

Stockholm University https://orcid.org/0000-0002-5302-1740

\section{Ondrej Gahura}

Institute of Parasitology, Biology Centre CAS https://orcid.org/0000-0002-2925-4763

\section{Alexander Muhleip}

Stockholm University https://orcid.org/0000-0002-1877-2282

\section{Carolina Hierro-Yap}

Institute of Parasitology, Biology Centre CAS

\section{Brian Panicucci}

Biology Centre

Minal Jain

Institute of Parasitology, Biology Centre CAS

\section{David Hollaus}

Institute of Parasitology, Biology Centre CAS https://orcid.org/0000-0001-7403-6434

\section{Martina Slapničková}

Institute of Parasitology, Biology Centre CAS

\section{Alena Zikova}

Biology Centre https://orcid.org/0000-0002-8686-0225

\section{Article}

Keywords:

Posted Date: December 30th, 2021

DOI: https://doi.org/10.21203/rs.3.rs-1196040/v1

License: (c) (1) This work is licensed under a Creative Commons Attribution 4.0 International License. Read Full License

Version of Record: A version of this preprint was published at Nature Communications on October 11th, 2022. See the published version at https://doi.org/10.1038/s41467-022-33588-z. 



\section{An ancestral interaction module promotes oligomerization in divergent mitochondrial ATP synthases}

Ondřej Gahura ${ }^{1, \dagger}$, Alexander Mühleip ${ }^{2, \dagger}$, Carolina Hierro-Yap ${ }^{1,3}$, Brian Panicucci ${ }^{1}$, Minal Jain ${ }^{1,3}$, David Hollaus ${ }^{3}$, Martina Slapničková ${ }^{1}$, Alena Zíková ${ }^{1,3,}$, Alexey Amunts ${ }^{2,4 *}$

${ }^{1}$ Institute of Parasitology, Biology Centre CAS, Ceske Budejovice, Czech Republic

${ }^{2}$ Science for Life Laboratory, Department of Biochemistry and Biophysics, Stockholm University, 17165 Solna, Sweden

${ }^{3}$ Faculty of Science, University of South Bohemia, Ceske Budejovice, Czech Republic

${ }^{4}$ Department of Physiology and Pharmacology, Karolinska Institutet, Stockholm, Sweden

*Correspondence to: azikova@paru.cas.cz; amunts@scilifelab.se

$6 \dagger$ These authors contributed equally to this work.

\section{Abstract}

0 Mitochondrial ATP synthase forms stable dimers arranged into oligomeric assemblies that 1 generate the inner-membrane curvature essential for efficient energy conversion. Here, we 2 report cryo-EM structures of the intact ATP synthase dimer from Trypanosoma brucei in ten 23 different rotational states. The model consists of 25 subunits, including nine lineage-specific, 24 as well as 36 lipids. The rotary mechanism is influenced by the divergent peripheral stalk, 25 conferring a greater conformational flexibility. Proton transfer in the lumenal half-channel 6 occurs via a chain of five ordered water molecules. The dimerization interface is formed by 7 subunit- $g$ that is critical for interactions but not for the catalytic activity. Although overall 8 dimer architecture varies among eukaryotes, we find that subunit- $g$ together with subunit-e 9 form an ancestral oligomerization motif, which is shared between the trypanosomal and 0 mammalian lineages. Therefore, our data defines the subunit-g/e module as a structural 1 component determining ATP synthase oligomeric assemblies. 
Mitochondrial ATP synthase consists of the soluble $\mathrm{F}_{1}$ and membrane-bound $\mathrm{F}_{\mathrm{o}}$ subcomplexes, and occurs in dimers that assemble into oligomers to induce the formation of inner-membrane folds, called cristae. The cristae are the sites for oxidative phosphorylation and energy conversion in eukaryotic cells. Dissociation of ATP synthase dimers into monomers results in the loss of native cristae architecture and impairs mitochondrial function ${ }^{1,2}$. While cristae morphology varies substantially between organisms from different lineages, ranging from flat lamellar in opisthokonts to coiled tubular in ciliates and discoidal in euglenozoans ${ }^{3}$, the mitochondrial ATP synthase dimers represent a universal occurrence to maintain the membrane shape ${ }^{4}$.

ATP synthase dimers of variable size and architecture, classified into types I to IV have recently been resolved by high-resolution cryo-EM studies. In the structure of the type-I ATP synthase dimer from mammals, the monomers are only weakly associated ${ }^{5,6}$, and in yeast insertions in the membrane subunits form tighter contacts ${ }^{7}$. The structure of the type-II ATP synthase dimer from the alga Polytomella sp. showed that the dimer interface is formed by phylum-specific components ${ }^{8}$. The type-III ATP synthase dimer from the ciliate Tetrahymena thermophila is characterized by parallel rotary axes, and a substoichiometric subunit, as well as multiple lipids were identified at the dimer interface, while additional protein components that tie the monomers together are distributed between the matrix, transmembrane, and lumenal regions ${ }^{9}$. The structure of the type-IV ATP synthase with native lipids from Euglena gracilis also showed that specific protein-lipid interactions contribute to the dimerization, and that the central and peripheral stalks interact with each other directly ${ }^{10}$. Finally, a unique apicomplexan ATP synthase dimerizes via 11 parasite-specific components that contribute $\sim 7000 \AA^{2}$ buried surface area ${ }^{11}$, and unlike all other ATP synthases, that assemble into rows, it associates in higher oligomeric states of pentagonal pyramids in the curved apical membrane regions. Together, the available structural data suggest a diversity of oligomerization, and it remains unknown whether common elements mediating these interactions exist or whether dimerization of ATP synthase occurred independently and multiple times in evolution ${ }^{4}$.

The ATP synthase of Trypanosoma brucei, a representative of kinetoplastids and an established medically important model organism causing the sleeping sickness, is highly divergent, exemplified by the pyramid-shaped $F_{1}$ head containing a phylum specific subunit ${ }^{12,13}$. The dimers are sensitive to the lack of cardiolipin ${ }^{14}$ and form short left-handed helical segments that extend across the membrane ridge of the discoidal cristae ${ }^{15}$. Uniquely among aerobic eukaryotes, the mammalian life cycle stage of $T$. brucei utilizes the reverse mode of ATP synthase, using the enzyme as a proton pump to maintain mitochondrial membrane potential at the expense of $\mathrm{ATP}^{16,17}$. In contrast, the insect stages of the parasite employ the ATPproducing forward mode of the enzyme ${ }^{18,19}$.

Given the conservation of the core subunits, the different nature of oligomerization and the ability to test structural hypotheses biochemically, we reasoned that investigation of the $T$. brucei ATP synthase structure and function would provide the missing evolutionary link to understand how the monomers interact to form physiological dimers. Here, we address this question by combining structural, functional and evolutionary analysis of the T. brucei ATP synthase dimer. 


\section{Cryo-EM structure of the T. brucei ATP synthase}

79 We purified ATP synthase dimers from cultured $T$. brucei procyclic trypomastigotes by affinity chromatography with a recombinant natural protein inhibitor $\mathrm{TbIF}_{1}{ }^{20}$, and subjected the sample to cryo-EM analysis (Extended Data Fig. 1 and 2). Using masked refinements, maps were obtained for the membrane region, the rotor, and the peripheral stalk. To describe the conformational space of the T. brucei ATP synthase, we resolved ten distinct rotary substates, which were refined to 3.5-4.3 $\AA$ resolution. Finally, particles with both monomers in rotational state 1 were selected, and the consensus structure of the dimer was refined to $3.2 \AA$ resolution (Extended Data Table 1, Extended Data Fig. 2).

Unlike the wide-angle architecture of dimers found in animals and fungi, the T. brucei ATP synthase displays an angle of $60^{\circ}$ between the two $\mathrm{F}_{1} / c$-ring subcomplexes. The model of the T. brucei ATP synthase includes all 25 different subunits, nine of which are lineage-specific (Fig. 1a, Supplementary Video 1, Extended Data Fig. 3). We named the subunits according to the previously proposed nomenclature ${ }^{21-23}$ (Extended Data Table 2). In addition, we identified and modeled 36 bound phospholipids, including 24 cardiolipins (Extended Data Fig. 4). Both detergents used during purification, $\mathrm{n}$-dodecyl $\beta$-D-maltoside ( $\beta$-DDM) and glyco-diosgenin (GDN) are also resolved in the periphery of the membrane region (Extended Data Fig. 5).

In the catalytic region, $\mathrm{F}_{1}$ is augmented by three copies of subunit $\mathrm{p} 18$, each bound to subunit$\alpha^{12,13}$. Our structure shows that $\mathrm{p} 18$ is involved in the unusual attachment of $\mathrm{F}_{1}$ to the peripheral stalk. The membrane region includes eight conserved $\mathrm{F}_{\mathrm{o}}$ subunits $(b, d, f, 8, i / j, k, e$, and $g)$ arranged around the central proton translocator subunit- $a$. We identified those subunits based on the structural similarity and matching topology to their yeast counterparts (Fig 2). For subunit- $b$, a single transmembrane helix superimposes well with $b \mathrm{H} 1$ from yeast and anchors the newly identified subunit-e and $-g$ to the $\mathrm{F}_{\mathrm{o}}$ (Fig 2a); a long helix $b \mathrm{H} 2$, which constitutes the central part of the peripheral stalk in other organisms is absent in T. brucei. The sequence of this highly reduced subunit- $b$ shows $18 \%$ identity and $40 \%$ similarity to $E$. gracilis subunit$b^{10}$, representing a diverged homolog (Extended Data Fig. 6). No alternative subunit- $b^{24}$ is 105 found in our structure.

106 The membrane region contains a peripheral subcomplex, formed primarily by the phylum107 specific ATPTB1,6,12 and ATPEG3 (Fig. 1b). It is separated from the conserved core by a 108 membrane-intrinsic cavity, in which nine bound cardiolipins are resolved (Fig. 1c), and the 109 C-terminus of ATPTB12 interacts with the lumenal $\beta$-barrel of the $c_{10}$-ring. In the cavity of the 110 decameric $c$-ring near the matrix side, 10 Arg66 c residues coordinate a ligand density, which 111 is consistent with a pyrimidine ribonucleoside triphosphate (Fig. 1d). We assign this density as 112 uridine-triphosphate (UTP), due to its large requirement in the mitochondrial RNA metabolism 113 of African trypanosomes being a substrate for post-transcriptional RNA editing ${ }^{25}$, and addition 114 of poly-uridine tails to gRNAs and $\operatorname{rRNAs}^{26,27}$, as well as due to low abundance of cytidine 115 triphosphate (CTP) $)^{28}$. The nucleotide base is inserted between two Arg82 cresidues, whereas 116 the triphosphate region is coordinated by another five Arg $82_{c}$ residues, with $T y r 79_{\delta}$ and $A s n 76_{\delta}$ 117 providing asymmetric coordination contacts. The presence of a nucleotide inside the $c$-ring is 
118 surprising, given the recent reports of phospholipids inside the $c$-rings in mammals ${ }^{5,6}$ and

119 ciliates $^{9}$, indicating that a range of different ligands can provide structural scaffolding.

120
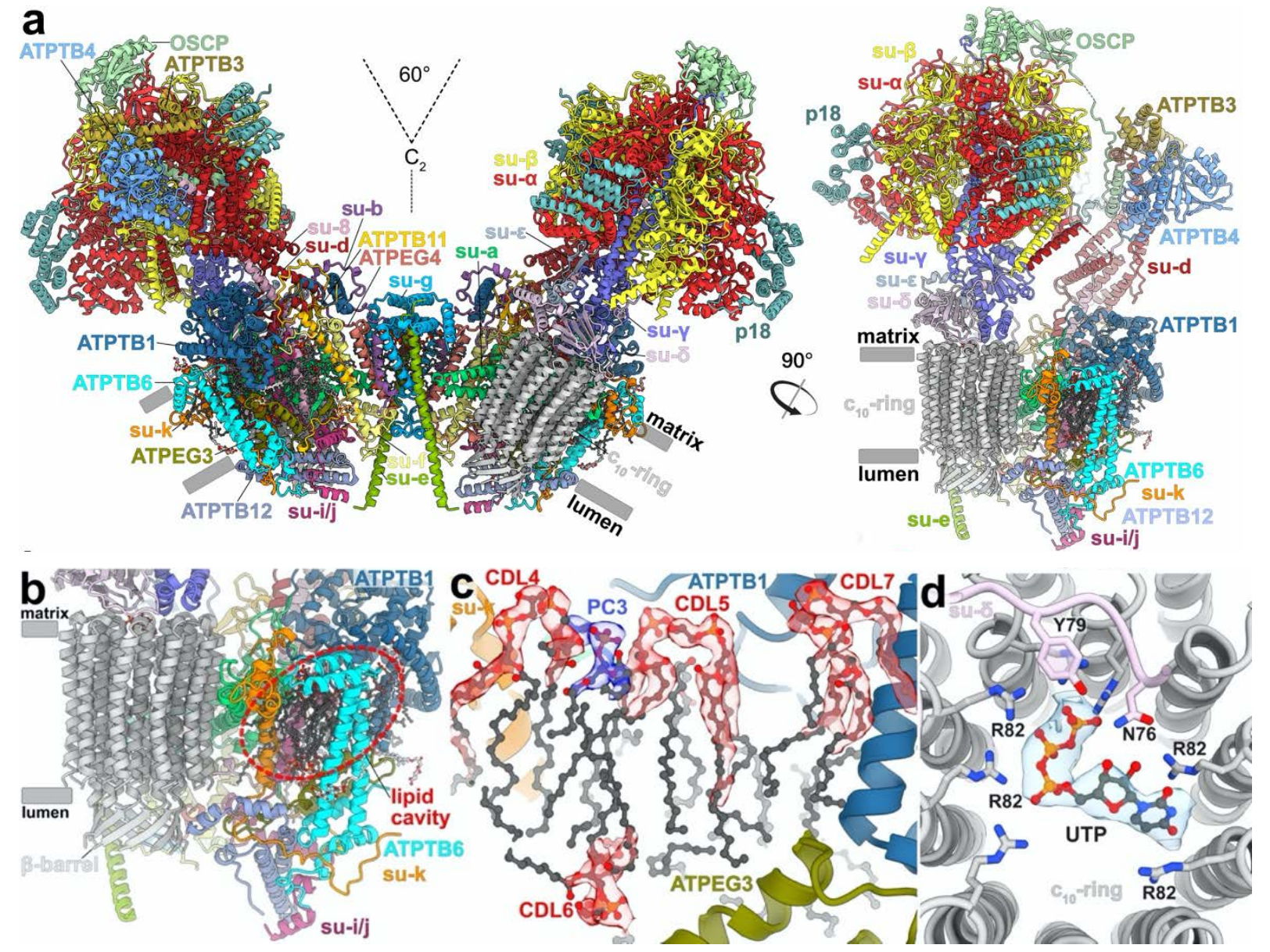

Fig. 1: The T. brucei ATP synthase structure with lipids and ligands.

a, Front and side views of the composite model with both monomers in rotational state 1 . The two $\mathrm{F}_{1} / c_{10}$-ring complexes, each augmented by three copies of the phylum-specific p 18 subunit, are tied together at a $60^{\circ}$-angle. The membrane-bound $\mathrm{F}_{\mathrm{o}}$ region displays a unique architecture and is composed of both conserved and phylum-specific subunits. $\mathbf{b}$, Side view of the $\mathrm{F}_{\mathrm{o}}$ region showing the lumenal interaction of the ten-stranded $\beta$-barrel of the $c$-ring (grey) with ATPTB12 (pale blue). The lipid-filled peripheral $F_{o}$ cavity is indicated. c, Close-up view of the bound lipids within the peripheral $F_{0}$ cavity with cryo-EM density shown. d, Top view into the decameric $c$-ring with a bound pyrimidine ribonucleoside triphosphate, assigned as UTP. Map density shown in transparent blue, interacting residues shown. 

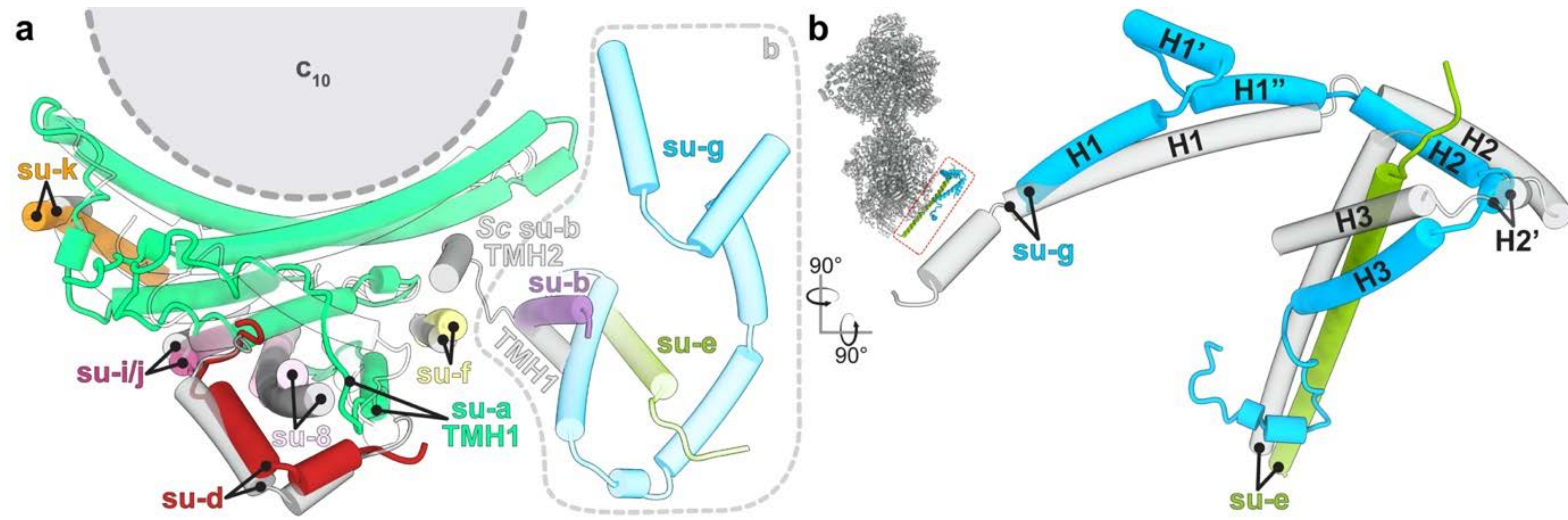

Fig. 2: Identification of conserved $F_{0}$ subunits.

134 a, Top view of the membrane region with $T$. brucei subunits (colored) overlaid with S. cerevisiae structure (gray transparent). Close structural superposition and matching topology allowed the assignment of conserved subunits based on matching topology and location. b, Superposition of subunits-e and $-g$ with their $S$. cerevisiae counterparts (PDB 6B2Z) confirms their identity.

\section{Peripheral stalk flexibility and distinct rotational states}

The trypanosomal peripheral stalk displays a markedly different architecture compared to its yeast and mammalian counterparts. In the opisthokont complexes, the peripheral stalk is organized around the long $b \mathrm{H} 2$, which extends from the membrane $\sim 15 \mathrm{~nm}$ into the matrix and attaches to OSCP at the top of $\mathrm{F}_{1}^{5,7}$. By contrast, $T$. brucei lacks the canonical $b \mathrm{H} 2$ and instead, helices 5-7 of divergent subunit- $d$ and the C-terminal helix of extended subunit- 8 bind to a Cterminal extension of OSCP at the apical part of the peripheral stalk (Fig. 3a). The interaction between OSCP and subunit- $d$ and -8 is stabilized by soluble ATPTB3 and ATPTB4. The peripheral stalk is rooted to the membrane subcomplex by a transmembrane helix of subunit8 , wrapped on the matrix side by helices $8-11$ of subunit- $d$. Apart from the canonical contacts at the top of $F_{1}$, the peripheral stalk is attached to the $F_{1}$ via a euglenozoa-specific $C$-terminal extension of OSCP, which contains a disordered linker and a terminal helix hairpin extending between the $\mathrm{F}_{1}$-bound $\mathrm{p} 18$ and subunits $-d$ and -8 of the peripheral stalk (Fig. 3a, Supplementary Videos 2,3). Another interaction of $F_{1}$ with the peripheral stalk occurs between the stacked C-terminal helices of subunit- $\beta$ and $-d$ (Fig. $3 b$ ), the latter of which structurally belongs to $F_{1}$ and is connected to the peripheral stalk via a flexible linker.

To assess whether the unusual peripheral stalk architecture influences the rotary mechanism, we analysed 10 classes representing different rotational states. The three main states (1-3) result from $\mathrm{a} \sim 120^{\circ}$ rotation of the central stalk subunit- $\gamma$, and we identified five (1a-1e), four (2a-2d) and one (3) classes of the respective main states. The rotor positions of the rotational states $1 \mathrm{a}$, $2 \mathrm{a}$ and 3 are related by steps of $117^{\circ}, 136^{\circ}$ and $107^{\circ}$, respectively. Throughout all the identified substeps of the rotational state 1 (classes 1 a to $1 \mathrm{e}$ ) the rotor turns by $\sim 33^{\circ}$, which corresponds approximately to the advancement by one subunit- $c$ of the $c_{10}$-ring. While rotating along with the rotor, the $\mathrm{F}_{1}$ headpiece lags behind, advancing by only $\sim 13^{\circ}$. During the following transition from $1 \mathrm{e}$ to $2 \mathrm{a}$, the rotor advances by $\sim 84^{\circ}$, whereas the $\mathrm{F}_{1}$ headpiece rotates $\sim 22^{\circ}$ in the opposite direction (Fig. 3c,d). This generates a counter-directional torque between the two motors, 
166

167

168

169

170

171

172

173

174

175

176

which is consistent with a power-stroke mechanism. Albeit with small differences in step size, this mechanism is consistent with a previous observation in the Polytomella ATP synthase ${ }^{8}$. However, due to its large, rigid peripheral stalk, the Polytomella ATP synthase mainly displays rotational substeps, whereas the Trypanosoma $\mathrm{F}_{1}$ also displays a tilting motion of $\sim 8^{\circ}$ revealed by rotary states 1 and 2 (Fig. 3e, Supplementary Video 2). The previously reported hinge motion between the $\mathrm{N}$ - and $\mathrm{C}$-terminal domains of $\mathrm{OSCP}^{8}$ is not found in our structures, instead, the conformational changes of the $\mathrm{F}_{1} / c_{10}$-ring subcomplex are accommodated by a $5^{\circ}$ bending of the apical part of the peripheral stalk. (Fig. 3e, Supplementary Videos 2,3). Together, the structural data indicate that the divergent peripheral stalk attachment confers greater conformational flexibility to the T. brucei ATP synthase.

177

178

179

180

181

182

183

184

185

186

187 a

$$
\text { C }
$$
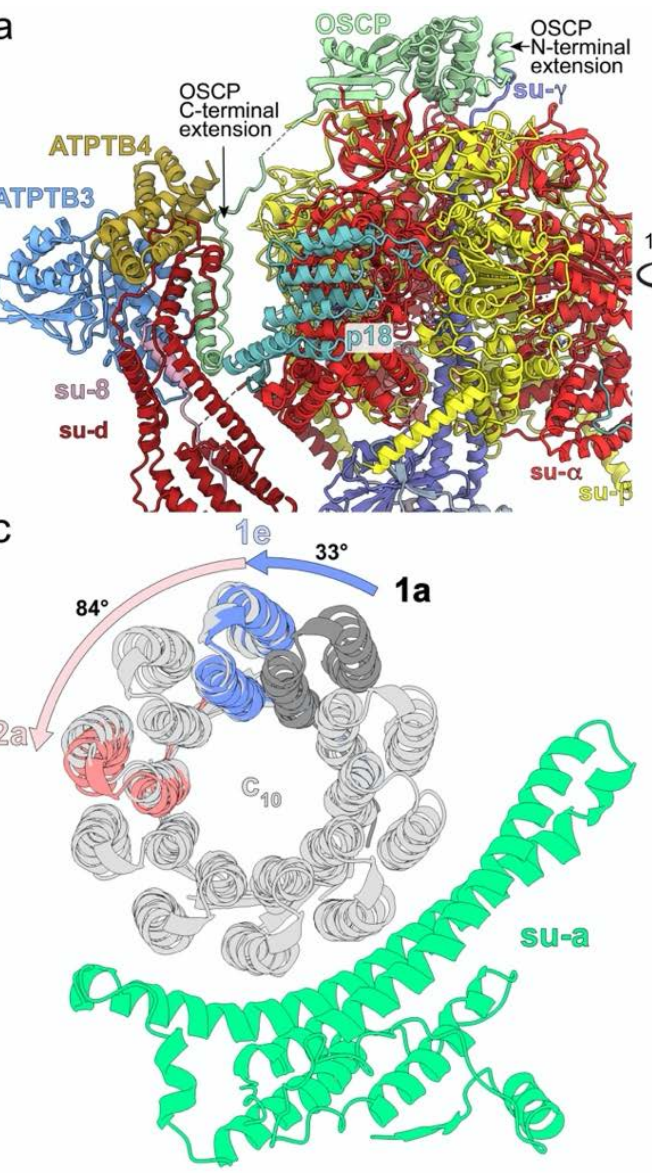
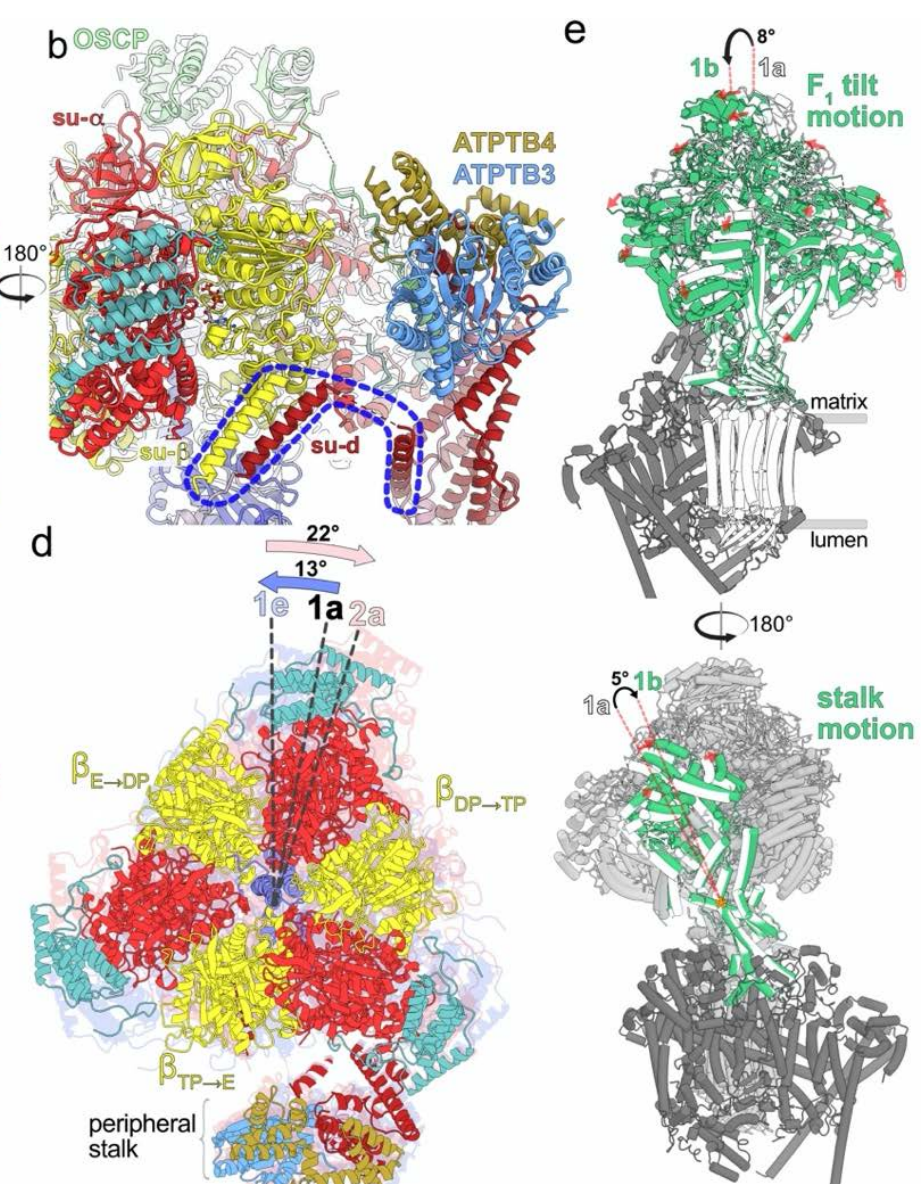

Fig. 3: A divergent peripheral stalk allows high flexibility during rotary catalysis. a, Nterminal OSCP extension provides a permanent central stalk attachment, while the C-terminal extension provides a phylum-specific attachment to the divergent peripheral stalk. b, The Cterminal helices of subunits $-\beta$ and $-d$ provide a permanent $\mathrm{F}_{1}$ attachment. c, Substeps of the $c$ ring during transition from rotational state 1 to 2 . $\mathbf{d}, \mathrm{F}_{1}$ motion accommodating steps shown in (c). After advancing along with the rotor to state $1 \mathrm{e}$, the $\mathrm{F}_{1}$ rotates in the opposite direction when transitioning to state $2 \mathrm{a}$. e, Tilting motion of $\mathrm{F}_{1}$ and accommodating bending of the peripheral stalk. 


\section{Lumenal proton half-channel is insulated by a lipid and contains ordered water molecules}

189 The mechanism of proton translocation involves sequential protonation of E102 of subunits-c, 190 rotation of the $c_{10}$-ring with neutralized E102c exposed to the phospholipid bilayer, and release 191 of protons on the other side of the membrane. The sites of proton binding and release are 192 separated by the conserved R146 contributed by the horizontal helix H5 of subunit- $a$ and are 193 accessible from the cristae lumen and mitochondrial matrix by aqueous half-channels (Fig. 4a).

194 Together, R146 and the adjacent N209 coordinate a pair of water molecules in between helices 195 H5 and H6 (Fig. 4b). A similar coordination has been observed in the Polytomella ATP 196 synthase ${ }^{8}$. The coordination of water likely restricts the R146 to rotamers that extend towards 197 the $c$-ring, with which it is thought to interact.

198 In our structure, the lumenal half-channel is filled with a network of resolved water densities, 199 ending in a chain of five ordered water molecules (W1-W5; Fig. 4c,d,e). The presence of 200 ordered water molecules in the aqueous channel is consistent with a Grotthuss-type mechanism 201 for proton transfer, which would not require long-distance diffusion of water molecules ${ }^{5}$. 202 However, because some distances between the observed water molecules are too large for 203 direct hydrogen bonding, proton transfer may involve both coordinated and disordered water 204 molecules. The distance of $7 \AA$ between the last resolved water (W1) and D202 ${ }_{a}$, the conserved 205 residue that is thought to transfer protons to the $c$-ring, is too long for direct proton transfer. 206 Instead, it may occur via the adjacent H155a. Therefore, our structure resolves individual 207 elements participating in proton transport (Fig. 4d,e).

208 The lumenal proton half-channel in the mammalian ${ }^{5,6}$ and apicomplexan ${ }^{11}$ ATP synthase is 209 lined by the transmembrane part of $b \mathrm{H} 2$, which is absent in T. brucei. Instead, the position of $210 b \mathrm{H} 2$ is occupied by a fully ordered phosphatidylcholine in our structure (PC1; Fig. 4a,c). 211 Therefore, a bound lipid replaces a proteinaceous element in the proton path. 
213 Fig. 4: The lumenal half-channel contains ordered water molecules and is confined by an

214 Fo-bound lipid. a, Subunit- $a$ (green) with the matrix (orange) and lumenal (light blue) 215 channels, and an ordered phosphatidylcholine (PC1; blue). E102 of the $c_{10}$-ring shown in grey.

216 b, Close-up view of the highly conserved R146 $a$ and $\mathrm{N} 209_{a}$, which coordinate two water molecules between helices H5-6a. c, Sideview of the lumenal channel with proton pathway

218 (light blue) and confining phosphatidylcholine (blue). d, Chain of ordered water molecules in the lumenal channel. Distances between the W1-W5 (red) are 5.2, 3.9, 7.3 and $4.8 \AA$, respectively. e, The ordered waters extend to H155a, which likely mediates the transfer of protons to D202a.

\section{Subunit- $g$ facilitates assembly of different ATP synthase oligomers}

224 Despite sharing a set of conserved $\mathrm{F}_{\mathrm{o}}$ subunits, the T. brucei ATP synthase dimer displays a markedly different dimer architecture compared to previously determined structures. First, its dimerization interface of $3,600 \AA^{2}$ is smaller than that of the E. gracilis type-IV $\left(10,000 \AA^{2}\right)$ and the T. thermophila type-III ATP synthases $\left(16,000 \AA^{2}\right)$. Second, unlike mammalian and fungal ATP synthase, in which the peripheral stalks extend in the plane defined by the two rotary axes, in our structure the monomers are rotated such that the peripheral stalks are offset laterally on the opposite sides of the plane. Due to the rotated monomers, this architecture is associated with a specific dimerization interface, where two subunit- $g$ copies interact homotypically on the $\mathrm{C}_{2}$ symmetry axis (Fig. 5a, Supplementary Video 1). Both copies of H1$2 \mathrm{~g}$ extend horizontally along the matrix side of the membrane, clamping against each other (Fig. 5c,e). This facilitates formation of contacts between an associated transmembrane helix 
of subunit-e with the neighbouring monomer via subunit- $a$ ' in the membrane, and $-f^{\prime}$ in the

236 lumen, thereby further contributing to the interface (Fig. 5b). Thus, the ATP synthase dimer is

237 assembled via the subunit- $e / g$ module. The $\mathrm{C}$-terminal part of the subunit- $e$ helix extends into

238 the lumen, towards the ten-stranded $\beta$-barrel of the $c$-ring (Extended Data Fig. 7a). The terminal

23923 residues are disordered with poorly resolved density connecting to the detergent plug of the

$240 \quad c$-ring $\beta$-barrel (Extended Data Fig. 7b). This resembles the lumenal C-terminus of subunit-e

241 in the bovine structure ${ }^{5}$, indicating a conserved interaction with the $c$-ring.

242 The $e / g$ module is held together by four bound cardiolipins in the matrix leaflet, anchoring it

243 to the remaining $F_{o}$ region (Fig. 5c). The head groups of the lipids are coordinated by polar and

244 charged residues with their acyl chains filling a central cavity in the membrane region at the

245 dimer interface (Fig 5c, Extended Data Fig. 4f). Cardiolipin binding has previously been

246 reported to be obligatory for dimerization in secondary transporters ${ }^{29}$ and the depletion of

247 cardiolipin synthase resulted in reduced levels of ATP synthase in bloodstream

248 trypanosomes ${ }^{14}$.

249 Interestingly, for yeasts, early blue native gel electrophoresis ${ }^{30}$ and subtomogram averaging

250 studies $^{2}$ suggested subunit- $g$ as potentially dimer-mediating, however the $e / g$ modules are

251 located laterally opposed on either side of the dimer long axis, in the periphery of the complex,

$252 \sim 8.5 \mathrm{~nm}$ apart from each other. Because the $e / g$ modules do not interact directly within the

253 yeast ATP synthase dimer, they have been proposed to serve as membrane-bending elements,

254 whereas the major dimer contacts are formed by subunit- $a$ and $-i / j^{7}$. In mammals, the $e / g$

255 module occupies the same position as in yeasts, forming the interaction between two diagonal

256 monomers in a tetramer ${ }^{5,6,31}$, as well as between parallel dimers ${ }^{32}$. The comparison with our

257 structure shows that the overall organization of the intra-dimeric trypanosomal and inter-

258 dimeric mammalian e/g module is structurally similar (Fig. 5d). Furthermore, kinetoplastid

259 parasites and mammals share conserved GXXXG motifs in subunit- $e^{33}$ and $-g$ (Extended Data

260 Fig. 8), which allow close interaction of their transmembrane helices (Fig. 5e), providing

261 further evidence for subunit homology. However, while the mammalian ATP synthase dimers

262 are arranged perpendicularly to the long axis of their rows along the edge of cristae ${ }^{34}$, the

$263 T$. brucei dimers on the rims of discoidal cristae are inclined $\sim 45^{\circ}$ to the row axis ${ }^{15}$. Therefore,

264 the $e / g$ module occupies equivalent positions in the rows of both evolutionary distant groups

265 (Fig. 5f and reference 32). 

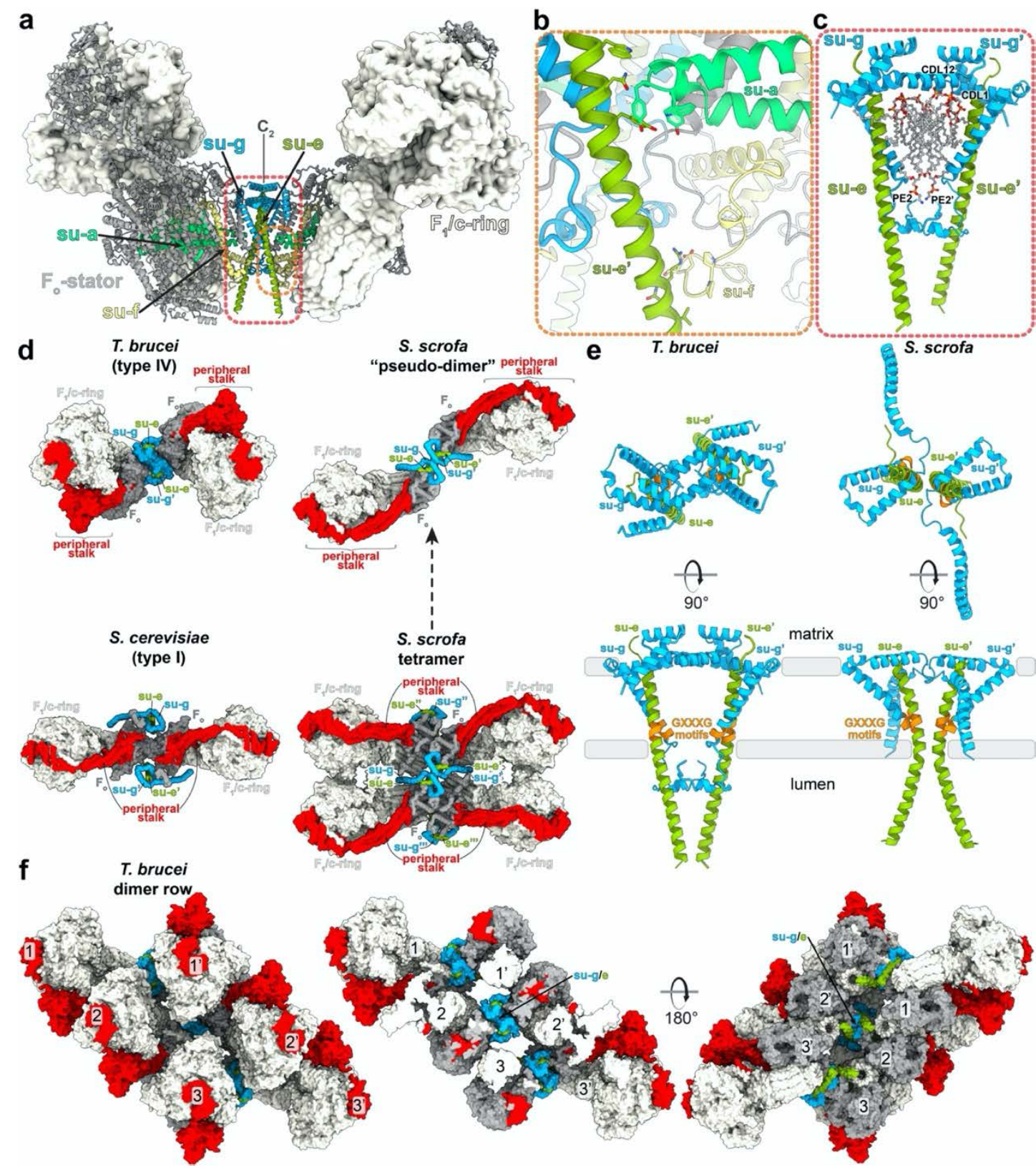

Fig. 5: The homotypic dimerization motif of subunit-g generates a conserved

268 oligomerization module. a, Side view with dimerising subunits colored. b,c, The dimer interface is constituted by (b) subunit- $e$ ' contacting subunit- $a$ in the membrane and subunit- $f$ in the lumen, (c) subunits $e$ and $g$ from both monomers forming a subcomplex with bound lipids. d, Subunit- $g$ and $-e$ form a dimerization motif in the trypanosomal (type-IV) ATP synthase dimer (this study), the same structural element forms the oligomerization motif in the porcine ATP synthase tetramer. The structural similarity of the pseudo-dimer (i.e., two diagonal monomers from adjacent dimers) in the porcine structure with the trypanosomal dimer suggests that type I and IV ATP synthase dimers have evolved through divergence from a common ancestor. e, The dimeric subunit-e/g structures are conserved in pig (PDB 6ZNA) and T. brucei (this work) and contain a conserved GXXXG motif (orange) mediating interaction of transmembrane helices. f, Models of the ATP synthase dimers fitted into subtomogram averages of short oligomers ${ }^{15}$ : matrix view, left; cut-through, middle, lumenal view, right (EMD-3560). 
a
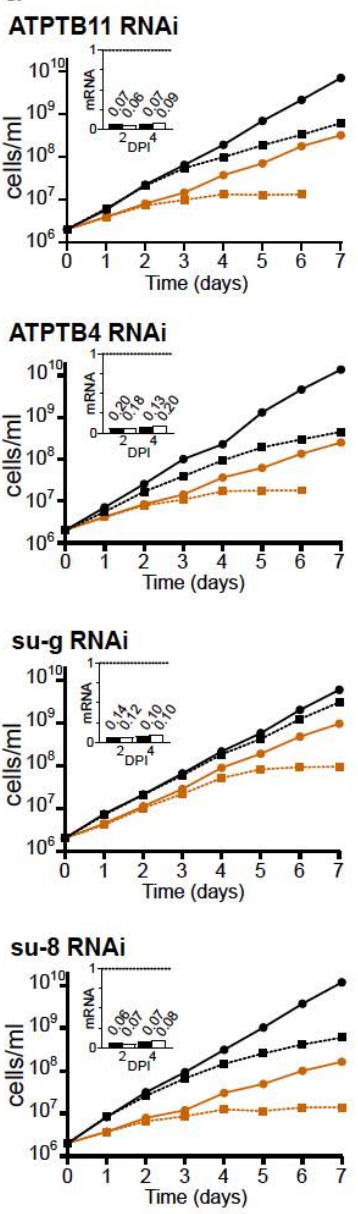

b
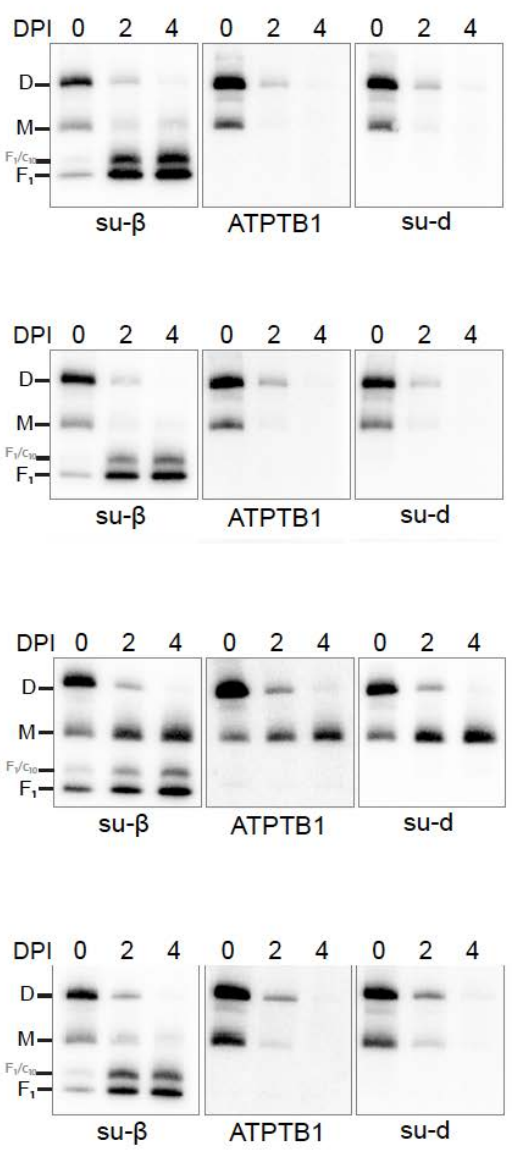

c
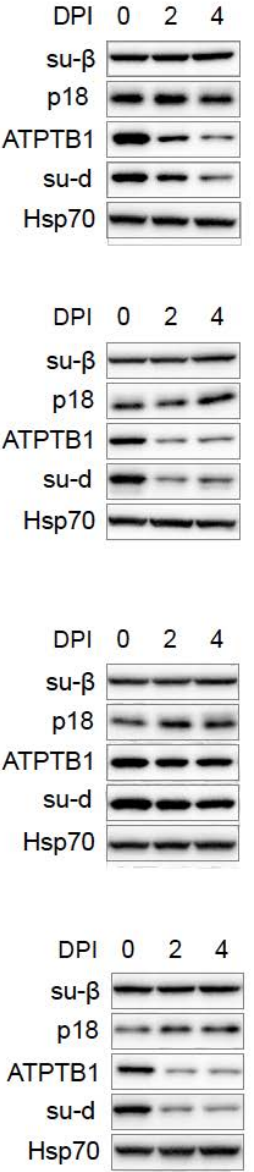

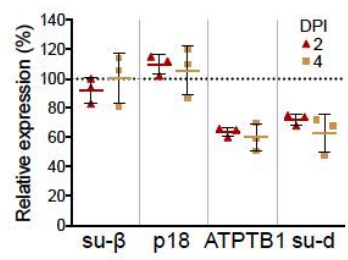

d
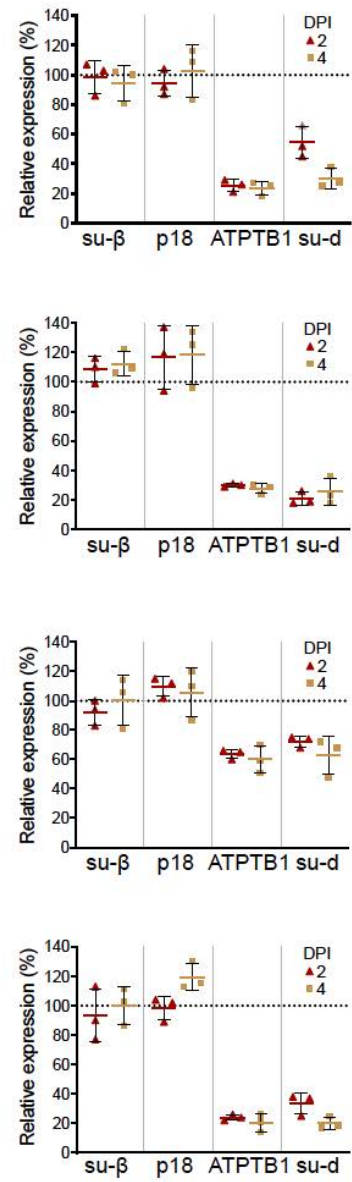

Fig. 6: RNAi knockdown of subunit-g results in monomerization of ATP synthase. a,

Growth curves of non-induced (solid lines) and tetracycline-induced (dashed lines) RNAi cell lines grown in the presence (black) or absence (brown) of glucose. The insets show relative levels of the respective target mRNA at indicated days post-induction (DPI) normalized to the levels of 18S rRNA (black bars) or $\beta$-tubulin (white bars). b, Immunoblots of mitochondrial lysates from indicated RNAi cell lines resolved by BN-PAGE probed with antibodies against indicated ATP synthase subunits. c, Representative immunoblots of whole cell lysates from indicated RNAi cell lines probed with indicated antibodies. d, Quantification of three replicates of immunoblots in (c). Values were normalized to the signal of the loading control Hsp70 and to non-induced cells. Plots show means with standard deviations (SD). 
293 To validate structural insights, we knocked down each individual $F_{0}$ subunit by inducible RNA 294 interference (RNAi). All target mRNAs dropped to 5-20\% of their original levels after two and four days of induction (Fig. 6a and Extended Data Fig. 9a). Western blot analysis of wholecell lysates resolved by denaturing electrophoresis revealed decreased levels of $F_{o}$ subunits ATPB1 and $-d$ suggesting that the integrity of the $\mathrm{F}_{\mathrm{o}}$ moiety depends on the presence of other $\mathrm{F}_{\mathrm{o}}$ subunits (Fig. 6c,d). Immunoblotting of mitochondrial complexes resolved by blue native polyacrylamide gel electrophoresis (BN-PAGE) with antibodies against $F_{1}$ and $F_{o}$ subunits revealed a strong decrease or nearly complete loss of dimeric and monomeric forms of ATP synthases four days after induction of RNAi of most subunits $(b, e, f, i / j, k, 8$, ATPTB3, ATPTB4, ATPTB6, ATPTB11, ATPTB12, ATPEG3 and ATPEG4), documenting an increased instability of the enzyme or defects in its assembly. Simultaneous accumulation in $\mathrm{F}_{1}$-ATPase, as observed by BN-PAGE, demonstrated that the catalytic moiety remains intact after the disruption of the peripheral stalk or the membrane subcomplex (Fig. 6b,c,d and Extended Data Fig. 9b).

In contrast to the other targeted $\mathrm{F}_{\mathrm{o}}$ subunits, the downregulation of subunit- $g$ with RNAi resulted in a specific loss of dimeric complexes with concomitant accumulation of monomers (Fig. 6b), indicating that it is required for dimerization, but not for the assembly and stability of the monomeric $\mathrm{F}_{1} \mathrm{~F}_{\mathrm{o}}$ ATP synthase units. Transmission electron microscopy of thin cell sections revealed that the ATP synthase monomerization in the subunit- $g^{\mathrm{RNAi}}$ cell line had the same effect on mitochondrial ultrastructure as nearly complete loss of monomers and dimers upon knockdown of subunit-8. Both cell lines exhibited decreased cristae counts and aberrant cristae morphology (Fig. 7a,b), including the appearance of round shapes reminiscent of structures detected upon deletion of subunit- $g$ or $-e$ in Saccharomyces cerevisiae ${ }^{1}$. These results indicate that monomerization prevents the trypanosomal ATP synthase from assembling into short helical rows on the rims of the discoidal cristae ${ }^{15}$, as has been reported for impaired oligomerization in counterparts from other eukaryotes ${ }^{2,35}$.

319 Despite the altered mitochondrial ultrastructure, the subunit- $g^{\mathrm{RNAi}}$ cells showed only a very mild growth phenotype, in contrast to all other RNAi cell lines that exhibited steadily slowed growth from day three to four after the RNAi induction (Fig. 7a, Extended Data Fig. 9a). This is consistent with the growth defects observed after the ablation of $F_{o}$ subunit ATPTB $1^{19}$ and $F_{1}$ subunits- $\alpha$ and $p 18^{12}$. Thus, the monomerization of ATP synthase upon subunit- $g$ ablation had only a negligible effect on the fitness of trypanosomes cultured in glucose-rich medium, in which ATP production by substrate level phosphorylation partially compensates for compromised oxidative phosphorylation ${ }^{36}$.

Measurement of oligomycin-sensitive ATP-dependent mitochondrial membrane polarization by safranin $\mathrm{O}$ assay in permeabilized cells showed that the proton pumping activity of the ATP synthase in the induced subunit- $g^{\text {RNAi }}$ cells is negligibly affected, demonstrating that the monomerized enzyme is catalytically functional. By contrast, RNAi downregulation of subunit-8, ATPTB4 and ATPTB11, and ATPTB1 resulted in a strong decline of the mitochondrial membrane polarization capacity, consistent with the loss of both monomeric and dimeric ATP synthase forms (Fig. 7c). Accordingly, knockdown of the same subunits resulted in inability to produce ATP by oxidative phosphorylation (Fig. 7d). However, upon subunit-g 
ablation the ATP production was affected only partially, confirming that the monomerized ATP synthase remains catalytically active. The $\sim 50 \%$ drop in ATP production of subunit- $g$ RNAi cells can be attributed to the decreased oxidative phosphorylation efficiency due to the impaired cristae morphology. Indeed, when cells were cultured in the absence of glucose, enforcing the need for oxidative phosphorylation, knockdown of subunit- $g$ results in a growth arrest, albeit one to two days later than knockdown of all other tested subunits (Fig. 6a). The data show that dimerization is critical when oxidative phosphorylation is the predominant source of ATP.
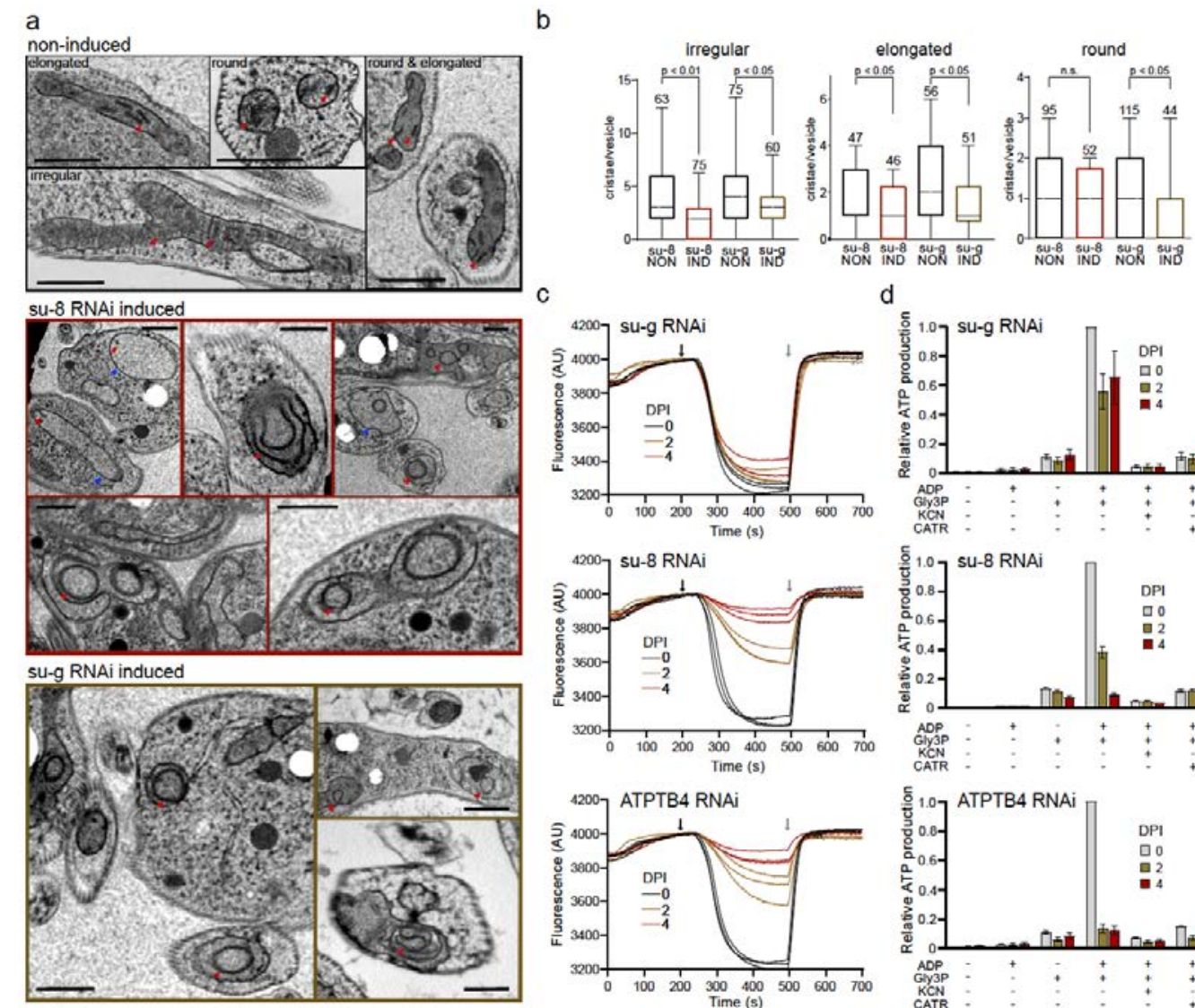

d

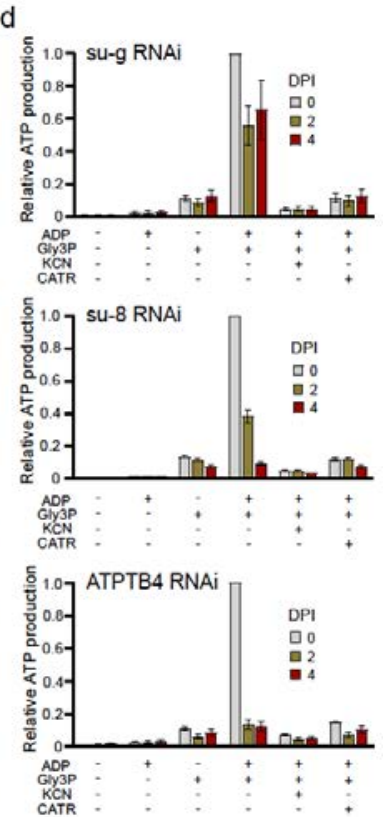

Fig. 7: Monomerization of ATP synthase by subunit-g knockdown results in aberrant mitochondrial ultrastructure but does not abolish catalytic activity. a, Transmission electron micrographs of sections of non-induced or 4 days induced RNAi cell lines. Mitochondrial membranes and cristae are marked with blue and red arrowheads, respectively. Top panel shows examples of irregular, elongated and round cross-sections of mitochondria quantified in (b). b. Cristae numbers per vesicle from indicated induced (IND) or non-induced (NON) cell lines counted separately in irregular, elongated and round mitochondrial crosssection. Boxes and whiskers show $25^{\text {th }}$ to $75^{\text {th }}$ and $5^{\text {th }}$ to $95^{\text {th }}$ percentiles, respectively. The numbers of analysed cross-sections are indicated for each data point. Unpaired t-test, $p$-values are shown. $\mathbf{c}$, Mitochondrial membrane polarization capacity of non-induced or RNAi-induced cell lines two and four DPI measured by Safranine O. Black and gray arrow indicate addition of ATP and oligomycin, respectively. d, ATP production in permeabilized non-induced (0) or RNAi-induced cells 2 and 4 DPI in the presence of indicated substrates and inhibitors. Error bars represent SD of three replicates. Gly3P, DL-glycerol phosphate; $K C N$, potassium cyanide; CATR, carboxyatractyloside 
360 Our structure of the mitochondrial ATP synthase dimer from the mammalian parasite T. brucei offers new insight into the mechanism of membrane shaping, rotary catalysis, and proton transfer. Considering that trypanosomes belong to an evolutionarily divergent group of Kinetoplastida, the ATP synthase dimer has several interesting features that differ from other dimer structures. The subunit- $b$ found in bacterial and other mitochondrial F-type ATP synthases appears to be highly reduced to a single transmembrane helix $b \mathrm{H} 1$. The long $b \mathrm{H} 2$, which constitutes the central part of the peripheral stalk in other organisms, and is also involved in the composition of the lumenal proton half-channel, is completely absent in T. brucei. Interestingly, the position of $b \mathrm{H} 2$ in the proton half channel is occupied by a fully ordered phosphatidylcholine molecule that replaces a well-conserved proteinaceous element in the proton path. Lack of the canonical $b \mathrm{H} 2$ also affects composition of the peripheral stalk in which the divergent subunit- $d$ and subunit- 8 binds directly to a C-terminal extension of OSCP, indicating a remodeled peripheral stalk architecture. The peripheral stalk contacts the $F_{1}$ headpiece at several positions conferring greater conformational flexibility to the ATP synthase.

Using the structural and functional data, we also identified a conserved structural element of the ATP synthase that is responsible for its multimerization. Particularly, subunit- $g$ is required for the dimerization, but dispensable for the assembly of the $F_{1} F_{o}$ monomers. Although the monomerized enzyme is catalytically competent, the inability to form dimers results in defective cristae structure, and consequently leads to compromised oxidative phosphorylation and cease of proliferation. The cristae-shaping properties of mitochondrial ATP synthase dimers are critical for sufficient ATP production by oxidative phosphorylation, but not for other mitochondrial functions, as demonstrated by the lack of growth phenotype of subunit $-g^{\mathrm{RNAi}}$ cells in the presence of glucose. Thus, trypanosomal subunit-g depletion strain represents an experimental tool to assess the roles of the enzyme's primary catalytic function and mitochondria-specific membrane-shaping activity, highlighting the importance of the latter for oxidative phosphorylation.

387 Based on our data and previously published structures, we propose an ancestral state with double rows of ATP synthase monomers connected by e/g modules longitudinally and by other $\mathrm{F}_{\mathrm{o}}$ subunits transversally. During the course of evolution, different pairs of adjacent ATP synthase monomer units formed stable dimers in individual lineages (Fig. 8). This gave rise to the highly divergent type-I and type-IV ATP synthase dimers with subunit-e/g modules serving either as oligomerization or dimerization motives, respectively. Because trypanosomes belong to the deep-branching eukaryotic supergroup Discoba, the proposed arrangement might have been present in the last eukaryotic common ancestor. Although sequence similarity of subunit$g$ is low and restricted to the single transmembrane helix, we found homologs of subunit- $g$ in addition to Opisthokonta and Discoba also in Archaeplastida and Amoebozoa, which represent other eukaryotic supergroups, thus supporting the ancestral role in oligomerization (Extended Data Fig. 8). Taken together, our analysis reveals that mitochondrial ATP synthases that display markedly diverged architecture share the ancestral structural module that promotes oligomerization. 


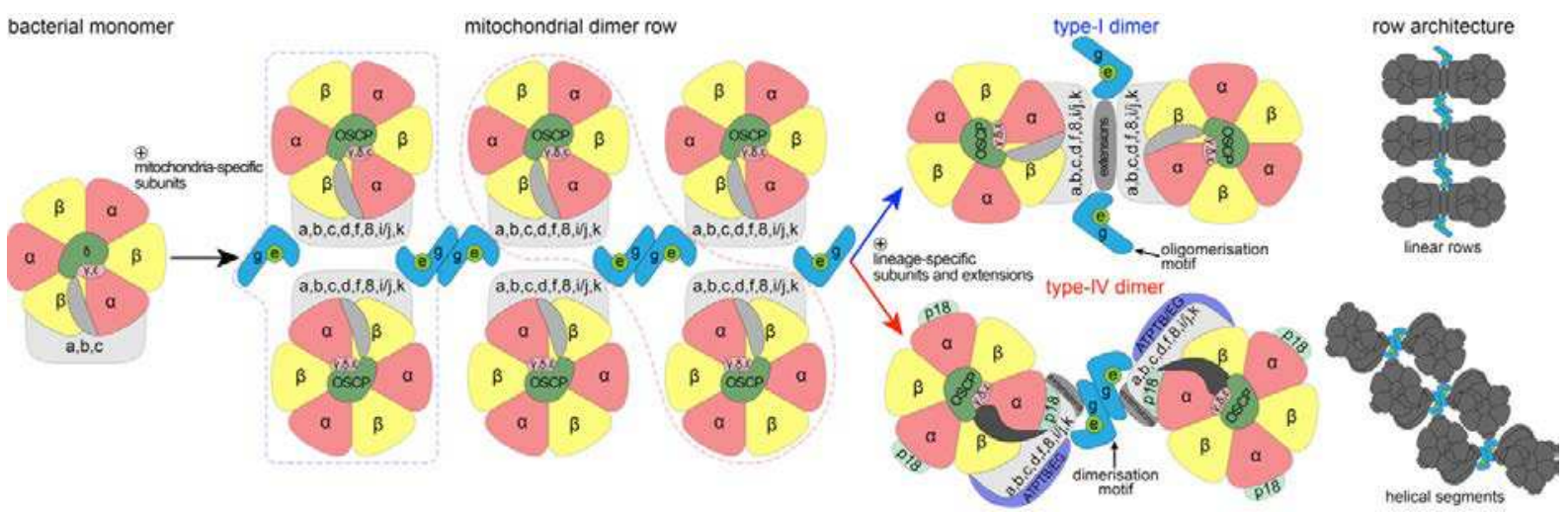

402

403

404

405

406

407

408

409

410

411

\section{Materials and Methods}

413 Cell culture and isolation of mitochondria

414 T. brucei procyclic strains were cultured in SDM-79 medium supplemented with 10\% (v/v) 415 fetal bovine serum. For growth curves in glucose-free conditions, cells were grown in SDM41680 medium with $10 \%$ dialysed FBS. RNAi cell lines were grown in presence of $2.5 \mu \mathrm{g} / \mathrm{ml}$ 417 phleomycin and $1 \mu \mathrm{g} / \mathrm{ml}$ puromycin. For ATP synthase purification, mitochondria were 418 isolated from the Lister strain 427 . Typically, $1.5 \times 10^{11}$ cells were harvested, washed in $20 \mathrm{mM}$ 419 sodium phosphate buffer pH 7.9 with $150 \mathrm{mM} \mathrm{NaCl}$ and $20 \mathrm{mM}$ glucose, resuspended in 420

Fig. 8: The subunit- $/ \mathrm{g}$ module is an ancestral oligomerization motif of ATP synthase. Schematic model of the evolution of type-I and IV ATP synthases. Mitochondrial ATP synthases are derived from a monomeric complex of proteobacterial origin. In a mitochondrial ancestor, acquisition of mitochondria-specific subunits, including the subunit-e/g module resulted in the assembly of ATP synthase double rows, the structural basis for cristae biogenesis. Through divergence, different ATP synthase architectures evolved, with the subunit-e/g module functioning as an oligomerization (type I) or dimerization (type IV) motif, resulting in distinct row assemblies between mitochondrial lineages. hypotonic buffer $1 \mathrm{mM}$ Tris-HCl pH 8.0, $1 \mathrm{mM}$ EDTA, and disrupted by 10 strokes in a 40-ml Dounce homogenizer. The lysis was stopped by immediate addition of sucrose to $0.25 \mathrm{M}$. Crude mitochondria were pelleted $\left(15 \mathrm{~min}\right.$ at $\left.16,000 \mathrm{xg}, 4^{\circ} \mathrm{C}\right)$, resuspended in $20 \mathrm{mM}$ Tris$\mathrm{HCl} \mathrm{pH}$ 8.0, $250 \mathrm{mM}$ sucrose, $5 \mathrm{mM} \mathrm{MgCl}_{2}, 0.3 \mathrm{mM} \mathrm{CaCl}_{2}$ and treated with $5 \mu \mathrm{g} / \mathrm{ml}$ DNase I. After $60 \mathrm{~min}$ on ice, one volume of the STE buffer $(20 \mathrm{mM}$ Tris- $\mathrm{HCl} \mathrm{pH} 8.0,250 \mathrm{mM}$ sucrose, $2 \mathrm{mM}$ EDTA) was added and mitochondria were pelleted (15 min at $16000 \mathrm{xg}, 4^{\circ} \mathrm{C}$ ). The pellet was resuspended in 60\% (v/v) Percoll in STE and loaded on six linear 10-35\% Percoll gradients in STE in polycarbonate tubes for SW28 rotor (Beckman). Gradients were centrifuged for $1 \mathrm{~h}$ at $24,000 \mathrm{rpm}, 4^{\circ} \mathrm{C}$. The middle phase containing mitochondrial vesicles $(15-20 \mathrm{ml}$ per tube) was collected, washed four times in the STE buffer, and pellets were snap-frozen in liquid nitrogen and stored at $-80^{\circ} \mathrm{C}$.

\section{Plasmid construction and generation of RNAi cell lines}

To downregulate ATP synthase subunits by RNAi, DNA fragments corresponding to individual target sequences were amplified by PCR from Lister 427 strain genomic DNA using 
forward and reverse primers extended with restriction sites XhoI\&KpnI and XbaI\&BamHI, respectively (Extended Data Table 3). Each fragment was inserted into the multiple cloning sites 1 and 2 of pAZ0055 vector, derived from pRP HYG-isL (courtesy of Sam Alsford) by replacement of hygromycine resistance gene with phleomycine resistance gene, with restriction

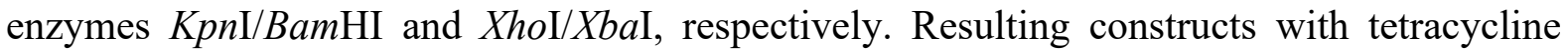
inducible T7 polymerase driven RNAi cassettes were linearized with NotI and transfected into a cell line derived from the Lister strain 427 by integration of the SmOx construct for expression of T7 polymerase and the tetracycline repressor ${ }^{37}$ into the $\beta$-tubulin locus. RNAi was induced in selected semi-clonal populations by addition of $1 \mu \mathrm{g} / \mathrm{ml}$ tetracycline and the downregulation of target mRNAs was verified by quantitative RT-PCR 2 and 4 days post induction. The total RNA isolated by an RNeasy Mini Kit (Qiagen) was treated with $2 \mu \mathrm{g}$ of DNase I, and then reverse transcribed to cDNA with TaqMan Reverse Transcription kit (Applied Biosciences). qPCR reactions were set with Light Cycler 480 SYBR Green I Master mix (Roche), $2 \mu \mathrm{l}$ of cDNA and $0.3 \mu \mathrm{M}$ primers (Extended Data Table 3), and run on LightCycler 480 (Roche). Relative expression of target genes was calculated using $-\Delta \Delta \mathrm{Ct}$ method with $18 \mathrm{~S}$ rRNA or $\beta$-tubulin as endogenous reference genes and normalized to noninduced cells.

\section{Denaturing and blue native polyacrylamide electrophoresis and immunoblotting}

Whole cell lysates for denaturing sodium dodecyl sulphate polyacrylamide electrophoresis (SDS-PAGE) were prepared from cells resuspended in PBS buffer (10 mM phosphate buffer, $130 \mathrm{mM} \mathrm{NaCl}, \mathrm{pH} 7.3$ ) by addition of $3 \mathrm{x}$ Laemmli buffer (150 mM Tris $\mathrm{pH} 6.8,300 \mathrm{mM}$ 1,4dithiothreitol, $6 \%(\mathrm{w} / \mathrm{v})$ SDS, $30 \%(\mathrm{w} / \mathrm{v})$ glycerol, $0.02 \%(\mathrm{w} / \mathrm{v})$ bromophenol blue) to final concentration of $1 \times 10^{7}$ cells in $30 \mu \mathrm{l}$. The lysates were boiled at $97^{\circ} \mathrm{C}$ for $10 \mathrm{~min}$ and stored at $-20^{\circ} \mathrm{C}$. For immunoblotting, lysates from $3 \times 10^{6}$ cells were separated on $4-20 \%$ gradient Trisglycine polyacrylamide gels (BioRad 4568094), electroblotted onto a PVDF membrane (Pierce 88518), and probed with respective antibodies (Extended Data Table 4). Membranes were incubated with the Clarity Western ECL substrate (BioRad 1705060EM) and chemiluminescence was detected on a ChemiDoc instrument (BioRad). Band intensities were quantified densitometrically using the ImageLab software. The levels of individual subunits were normalized to the signal of mtHsp70.

Blue native PAGE (BN-PAGE) was performed as described earlier ${ }^{12}$ with following modifications. Crude mitochondrial vesicles from $2.5 \times 10^{8}$ cells were resuspended in $40 \mu 1$ of Solubilization buffer A ( $2 \mathrm{mM} \varepsilon$-aminocaproic acid (ACA), $1 \mathrm{mM}$ EDTA, $50 \mathrm{mM} \mathrm{NaCl}, 50$ $\mathrm{mM}$ Bis-Tris/HCl, $\mathrm{pH} 7.0)$ and solubilized with $2 \%(\mathrm{w} / \mathrm{v})$ dodecylmaltoside ( $\beta$-DDM) for $1 \mathrm{~h}$ on ice. Lysates were cleared at $16,000 \mathrm{~g}$ for $30 \mathrm{~min}$ at $4^{\circ} \mathrm{C}$ and their protein concentration was estimated using bicinchoninic acid assay. For each time point, a volume of mitochondrial lysate corresponding to $4 \mu \mathrm{g}$ of total protein was mixed with $1.5 \mu \mathrm{l}$ of loading dye $(500 \mathrm{mM} \mathrm{ACA}$, $5 \%(\mathrm{w} / \mathrm{v})$ Coomassie Brilliant Blue G-250) and 5\% (w/v) glycerol and with $1 \mathrm{M} \mathrm{ACA}$ until a final volume of $20 \mu \mathrm{l} / \mathrm{well}$, and resolved on a native PAGE 3-12\% Bis-Tris gel (Invitrogen). After the electrophoresis $\left(3 \mathrm{~h}, 140 \mathrm{~V}, 4^{\circ} \mathrm{C}\right)$, proteins were transferred by electroblotting onto a 
PVDF membrane $\left(2 \mathrm{~h}, 100 \mathrm{~V}, 4^{\circ} \mathrm{C}\right.$, stirring), followed by immunodetection with an appropriate antibody (Extended Data Table 4).

\section{Mitochondrial membrane polarization measurement}

The capacity to polarize mitochondrial membrane was determined fluorometrically employing safranin $O$ dye (Sigma S2255) in permeabilized cells. For each sample, $2 \times 10^{7}$ cells were harvested and washed with ANT buffer $(8 \mathrm{mM} \mathrm{KCl}, 110 \mathrm{mM}$ K-gluconate, $10 \mathrm{mM} \mathrm{NaCl}, 10$ $\mathrm{mM}$ free-acid Hepes, $10 \mathrm{mM} \mathrm{K}_{2} \mathrm{HPO}_{4}, 0.015 \mathrm{mM}$ EGTA potassium salt, $10 \mathrm{mM}$ mannitol, 0.5 $\mathrm{mg} / \mathrm{ml}$ fatty acid-free BSA, $1.5 \mathrm{mM} \mathrm{MgCl}_{2}, \mathrm{pH} 7.25$ ). The cells were permeabilized by $8 \mu \mathrm{M}$ digitonin in $2 \mathrm{ml}$ of ANT buffer containing $5 \mu \mathrm{M}$ safranin O. Fluorescence was recorded for $700 \mathrm{~s}$ in a Hitachi F-7100 spectrofluorimeter (Hitachi High Technologies) at a 5-Hz acquisition rate, using $495 \mathrm{~nm}$ and $585 \mathrm{~nm}$ excitation and emission wavelengths, respectively. $1 \mathrm{mM}$ ATP (PanReac AppliChem A1348,0025) and $10 \mu \mathrm{g} / \mathrm{ml}$ oligomycin (Sigma O4876) were added after $230 \mathrm{~s}$ and $500 \mathrm{~s}$, respectively. Final addition of the uncoupler SF 6847 (250 nM; Enzo Life Sciences BML-EI215-0050) served as a control for maximal depolarization. All experiments were performed at room temperature and constant stirring.

\section{ATP production assay}

ATP production in digitonin-isolated mitochondria was performed as described previously ${ }^{38}$. Briefly, $1 \times 10^{8}$ cells per time point were lysed in SoTE buffer ( $600 \mathrm{mM}$ sorbitol, $2 \mathrm{mM}$ EDTA, $20 \mathrm{mM}$ Tris-HCl, $\mathrm{pH} 7.75$ ) containing $0.015 \%(\mathrm{w} / \mathrm{v})$ digitonin for $5 \mathrm{~min}$ on ice. After centrifugation $\left(3 \mathrm{~min}, 4,000 \mathrm{~g}, 4^{\circ} \mathrm{C}\right)$, the soluble cytosolic fraction was discarded and the organellar pellet was resuspended in $75 \mu \mathrm{l}$ of ATP production assay buffer ( $600 \mathrm{mM}$ sorbitol, $10 \mathrm{mM} \mathrm{MgSO}$, $15 \mathrm{mM}$ potassium phosphate buffer $\mathrm{pH}$ 7.4, $20 \mathrm{mM}$ Tris- $\mathrm{HCl} \mathrm{pH}$ 7.4, 2.5 $\mathrm{mg} / \mathrm{ml}$ fatty acid-free BSA). ATP production was induced by addition of $20 \mathrm{mM}$ DL-glycerol phosphate (sodium salt) and $67 \mu \mathrm{M}$ ADP. Control samples were preincubated with the inhibitors potassium cyanide $(1 \mathrm{mM})$ and carboxyatractyloside $(6.5 \mu \mathrm{M})$ for $10 \mathrm{~min}$ at room temperature. After $30 \mathrm{~min}$ at room temperature, the reaction was stopped by addition of $1.5 \mu 1$ of $70 \%$ perchloric acid. The concentration of ATP was estimated using the Roche ATP Bioluminescence Assay Kit HS II in a Tecan Spark plate reader. The luminescence values of the RNAi induced samples were normalized to that of the corresponding noninduced sample.

\section{Thin sectioning and transmission electron microscopy}

The samples were centrifuged and pellet was transferred to the specimen carriers which were completed with 20\% BSA and immediately frozen using high pressure freezer Leica EM ICE (Leica Microsystems). Freeze substitution was performed in the presence of $2 \%$ osmium tetroxide diluted in $100 \%$ acetone at $-90^{\circ} \mathrm{C}$. After $96 \mathrm{~h}$, specimens were warmed to $-20^{\circ} \mathrm{C}$ at a slope $5{ }^{\circ} \mathrm{C} / \mathrm{h}$. After the next $24 \mathrm{~h}$, the temperature was increased to $3^{\circ} \mathrm{C}\left(3^{\circ} \mathrm{C} / \mathrm{h}\right)$. At room temperature, samples were washed in acetone and infiltrated with $25 \%, 50 \%, 75 \%$ acetone/resin EMbed 812 (EMS) mixture $1 \mathrm{~h}$ at each step. Finally, samples were infiltrated in $100 \%$ resin and polymerized at $60^{\circ} \mathrm{C}$ for $48 \mathrm{~h}$. Ultrathin sections $(70 \mathrm{~nm})$ were cut using a 
517 diamond knife, placed on copper grids and stained with uranyl acetate and lead citrate. TEM

518 micrographs were taken with Mega View III camera (SIS) using a JEOL 1010 TEM operating 519 at an accelerating voltage of $80 \mathrm{kV}$.

$521 \quad$ Purification of T. brucei ATP synthase dimers

522 Mitochondria from $3 \times 10^{11}$ cells were lysed by $1 \%(\mathrm{w} / \mathrm{v}) \beta$-DDM in $60 \mathrm{ml}$ of $20 \mathrm{mM}$ Bis-tris 523 propane $\mathrm{pH} 8.0$ with $10 \%$ glycerol and EDTA-free Complete protease inhibitors (Roche) for $52420 \mathrm{~min}$ at $4^{\circ} \mathrm{C}$. The lysate was cleared by centrifugation at $30,000 \mathrm{xg}$ for $20 \mathrm{~min}$ at $4^{\circ} \mathrm{C}$ and 525 adjusted to $\mathrm{pH} 6.8$ by drop-wise addition of $1 \mathrm{M} 3-(\mathrm{N}$-morpholino) propanesulfonic acid $\mathrm{pH}$ 526 5.9. Recombinant $\mathrm{TbIF}_{1}$ without dimerization region, whose affinity to $\mathrm{F}_{1}$-ATPase was 527 increased by N-terminal truncation and substitution of tyrosine 36 with tryptophan ${ }^{20}$, with a C528 terminal glutathione S-transferase (GST) tag ( TbIF $\left._{1}(9-64)-Y 36 W-G S T\right)$ was added in 529 approximately 10 -fold molar excess over the estimated content of ATP synthase. Binding of $530 \mathrm{TbIF}_{1}$ was facilitated by addition of neutralized $2 \mathrm{mM}$ ATP with $4 \mathrm{mM}$ magnesium sulphate. 531 After $5 \mathrm{~min}$, sodium chloride was added to $100 \mathrm{mM}$, the lysate was filtered through a $0.2 \mu \mathrm{m}$ syringe filter and immediately loaded on $5 \mathrm{ml}$ GSTrap HP column (Cytiva) equilibrated in 20 mM Bis-Tris-Propane $\mathrm{pH} 6.8$ binding buffer containing $0.1 \%(\mathrm{w} / \mathrm{v})$ glyco-diosgenin (GDN; Avanti Polar Lipids), $10 \%$ (v/v) glycerol, $100 \mathrm{mM}$ sodium chloride, $1 \mathrm{mM}$ tris(2carboxyethyl)phosphine (TCEP), $1 \mathrm{mM}$ ATP, $2 \mathrm{mM}$ magnesium sulphate, $15 \mu \mathrm{g} / \mathrm{ml}$ cardiolipin, $50 \mu \mathrm{g} / \mathrm{ml}$ 1-palmitoyl-2-oleoyl-sn-glycero-3-phosphocholine (POPC), $25 \mu \mathrm{g} / \mathrm{ml} \mathrm{1-}$ palmitoyl-2-oleoyl-sn-glycero-3-phosphoethanolamine (POPE) and $10 \mu \mathrm{g} / \mathrm{ml}$ 1-palmitoyl-2oleoyl-sn-glycero-3-[phospho-rac-(1-glycerol)] (POPG). All phospholipids were purchased from Avanti Polar Lipids (catalog numbers 840012C, 850457C, 850757C and 840757, respectively). ATP synthase was eluted with a gradient of $20 \mathrm{mM}$ reduced glutathione in Tris $\mathrm{pH} 8.0$ buffer containing the same components as the binding buffer. Fractions containing ATP synthase were pooled and concentrated to $150 \mu \mathrm{l}$ on Vivaspin centrifugal concentrator with 30 $\mathrm{kDa}$ molecular weight cut-off. The sample was fractionated by size exclusion chromatography on a Superose 6 Increase 3.2/300 GL column (Cytiva) equilibrated in a buffer containing 20 $\mathrm{mM}$ Tris $\mathrm{pH} 8.0,100 \mathrm{mM}$ sodium chloride, $2 \mathrm{mM}$ magnesium chloride, $0.1 \%$ (w/v) GDN, $3.75 \mu \mathrm{g} / \mathrm{ml}$ cardiolipin, $12.5 \mu \mathrm{g} / \mathrm{ml}$ POPC, $6.25 \mu \mathrm{g} / \mathrm{ml}$ POPE and $2.5 \mu \mathrm{g} / \mathrm{ml}$ POPG at 0.03 $\mathrm{ml} / \mathrm{min}$. Fractions corresponding to ATP synthase were pooled, supplemented with $0.05 \%$ $(\mathrm{w} / \mathrm{v}) \beta$-DDM that we and others experimentally found to better preserve dimer assemblies in cryo-EM ${ }^{39}$, and concentrated to $50 \mu 1$.

\section{Preparation of cryo-EM grids and data collection}

552 Samples were vitrified on glow-discharged Quantifoil R1.2/1.3 Au 300-mesh grids after blotting for $3 \mathrm{sec}$, followed by plunging into liquid ethane using a Vitrobot Mark IV. 5,199 movies were collected using EPU 1.9 on a Titan Krios (ThermoFisher Scientific) operated at $300 \mathrm{kV}$ at a nominal magnification of $165 \mathrm{kx}(0.83 \AA /$ pixel $)$ with a Quantum K2 camera (Gatan) using a slit width of $20 \mathrm{eV}$. Data was collected with an exposure rate of 3.6 electrons/px/s, a total exposure of 33 electrons $/ \AA^{2}$ and 20 frames per movie. 
560 Image processing was performed within the Scipion 2 framework $^{40}$, using RELION-3.0 unless

561 specified otherwise. Movies were motion-corrected using the RELION implementation of the 562 MotionCor2. 294,054 particles were initially picked using reference-based picking in 563 Gautomatch (http://www.mrc-lmb.cam.ac.uk/kzhang/Gautomatch) and Contrast-transfer 564 function parameters were using $\mathrm{GCTF}^{41}$. Subsequent image processing was performed in 565 RELION-3.0 and 2D and 3D classification was used to select 100,605 particles, which were 566 then extracted in an unbinned 560-pixel box (Fig. S1). An initial model of the ATP synthase 567 dimer was obtained using de novo 3D model generation. Using masked refinement with applied $568 \mathrm{C}_{2}$ symmetry, a 2.7- $\AA$ structure of the membrane region was obtained following per-particle $569 \mathrm{CTF}$ refinement and Bayesian polishing. Following $\mathrm{C}_{2}$-symmetry expansion and signal 570 subtraction of one monomer, a $3.7 \AA$ map of the peripheral stalk was obtained. Using 3D 571 classification $(\mathrm{T}=100)$ of aligned particles, with a mask on the $\mathrm{F}_{1 / \mathcal{C}}$-ring region, 10 different 572 rotational substates were then separated and maps at 3.5-4.3 $\AA$ resolution were obtained using $5733 \mathrm{D}$ refinement. The authors note that the number of classes identified in this study likely 574 reflects the limited number of particles, rather than the complete conformational space of the 575 complex. By combining particles from all states belonging to main rotational state 1, a 3.7- $\AA$ 576 map of the rotor and a 3.2-A consensus map of the complete ATP synthase dimer with both rotors in main rotational state 1 were obtained.

Model building, refinement and data visualization

580 An initial atomic model of the static $F_{0}$ membrane region was built automatically using 581 Bucaneer ${ }^{42}$. Subunits were subsequently assigned directly from the cryo-EM map, 15 of them 582 corresponding to previously identified T. brucei ATP synthase subunits ${ }^{21}$, while three subunits 583 (ATPTB14, ATPEG3, ATPEG4) were newly identified using BLAST searches. Manual model 584 building was performed in Coot using the T. brucei $\mathrm{F}_{1}$ (PDB 6F5D) ${ }^{13}$ and homology models ${ }^{43}$ 585 of the E. gracilis OSCP and $c$-ring (PDB 6TDU) ${ }^{10}$ as starting models. Ligands were manually 586 fitted to the map and restraints were generated by the GRADE server 587 (http://grade.globalphasing.org). Real-space refinement was performed in PHENIX using auto588 sharpened, local-resolution-filtered maps of the membrane region, peripheral stalk tip, $589 c$-ring/central stalk and $\mathrm{F}_{1} \mathrm{~F}_{\mathrm{o}}$ monomers in different rotational states, respectively, using 590 secondary structure restraints. Model statistics were generated using MolProbity ${ }^{44}$ and 591 EMRinger ${ }^{44} 5$ Finally, the respective refined models were combined into a composite ATP 592 synthase dimer model and real-space refined against the local-resolution-filtered consensus 593 ATP synthase dimer map with both monomers in rotational state 1, applying reference 594 restraints. Figures of the structures were prepared using ChimeraX ${ }^{46}$, the proton half-channels 595 were traced using HOLLOW ${ }^{47}$.

\section{Data availability}

598 The atomic coordinates have been deposited in the Protein Data Bank (PDB) and are available 599 under the accession codes: XXXX (membrane-region), XXXX (peripheral stalk), XXXX 600 (rotor), XXXX (F1Fo dimer), XXXX (rotational state 1a), XXXX (rotational state 1b), XXXX 
(rotational state 1c), XXXX (rotational state 1d), XXXX (rotational state 1e), XXXX (rotational state 2a), XXXX (rotational state 2b), XXXX (rotational state 2c), XXXX (rotational state 2d), XXXX (rotational state 3). The local resolution filtered cryo-EM maps, half maps, masks and FSC-curves have been deposited in the Electron Microscopy Data Bank with the accession codes: EMD-XXXX (membrane-region), EMD-XXXX (peripheral stalk), EMD-XXXX (rotor), EMD-XXXX ( $F_{1} F_{0}$ dimer), EMD-XXXX (rotational state 1a), EMDXXXX (rotational state 1b), EMD-XXXX (rotational state 1c), EMD-XXXX (rotational state 1d), EMD-XXXX (rotational state 1e), EMD-XXXX (rotational state 2a), EMD-XXXX (rotational state 2b), EMD-XXXX (rotational state 2c), EMD-XXXX (rotational state 2d), EMD-XXXX (rotational state 3).

\section{Acknowledgements}

We are grateful to Sir John E. Walker and Martin Montgomery for invaluable assistance with ATP synthase purification in the initial stage of the project. We acknowledge cryo-electron microscopy and tomography core facility of CIISB, Instruct-CZ Centre, supported by MEYS CR (LM2018127). This work was supported by the Czech Science Foundation grants number 18-17529S to A.Z. and 20-04150Y to O.G. and by European Regional Development Fund (ERDF) and Ministry of Education, Youth and Sport (MEYS) project CZ.02.1.01/0.0/0.0/16_019/0000759 to A.Z., Swedish Foundation for Strategic Research (FFL15:0325), Ragnar Söderberg Foundation (M44/16), European Research Council (ERC2018-StG-805230), Knut and Alice Wallenberg Foundation (2018.0080), and EMBO Young Investigator Programme to A.A.

\section{Author contributions}

A.Z. and A.A. conceived and designed the work. O.G. prepared the sample for cryo-EM. O.G. and A.M. performed initial screening. A.M. processed the cryo-EM data and built the model. O.G., A.M. and A.A. analyzed the structure. B.P., C.H.Y., M.J., M.S., O.G. and A.Z. performed biochemical analysis. O.G., A.M., A.A. and A.Z. interpreted the data. O.G., A.M., A.A. and A.Z. wrote and revised the manuscript. All authors contributed to the analysis and approved the final version of the manuscript.

\section{Competing interests}

The authors declare no competing interests.

\section{References}

1. Paumard, P. et al. The ATP synthase is involved in generating mitochondrial cristae morphology. EMBO J 21, 221-30 (2002).

2. Davies, K.M., Anselmi, C., Wittig, I., Faraldo-Gomez, J.D. \& Kuhlbrandt, W. Structure of the yeast $\mathrm{F}_{1} \mathrm{~F}_{\mathrm{o}}$-ATP synthase dimer and its role in shaping the mitochondrial cristae. Proc Natl Acad Sci U S A 109, 13602-7 (2012). 
641

642

643

644

645

646

647

648

649

650

651

652

653

654

655

656

657

658

659

660

661

662

663

664

665

666

667

668

669

670

671

672

673

674

675

676

677

678

679

680

681

682

3. Panek, T., Elias, M., Vancova, M., Lukes, J. \& Hashimi, H. Returning to the Fold for Lessons in Mitochondrial Crista Diversity and Evolution. Curr Biol 30, R575-R588 (2020).

4. Kuhlbrandt, W. Structure and Mechanisms of F-Type ATP Synthases. Annu Rev Biochem 88, 515-549 (2019).

5. Spikes, T.E., Montgomery, M.G. \& Walker, J.E. Structure of the dimeric ATP synthase from bovine mitochondria. Proc Natl Acad Sci US A 117, 23519-23526 (2020).

6. Pinke, G., Zhou, L. \& Sazanov, L.A. Cryo-EM structure of the entire mammalian Ftype ATP synthase. Nat Struct Mol Biol 27, 1077-1085 (2020).

7. Guo, H., Bueler, S.A. \& Rubinstein, J.L. Atomic model for the dimeric $F_{0}$ region of mitochondrial ATP synthase. Science 358, 936-940 (2017).

8. Murphy, B.J. et al. Rotary substates of mitochondrial ATP synthase reveal the basis of flexible $\mathrm{F}_{1}-\mathrm{F}_{\mathrm{o}}$ coupling. Science 364, eaaw9128 (2019).

9. Flygaard, R.K., Mühleip, A., Tobiasson, V. \& Amunts, A. Type III ATP synthase is a symmetry-deviated dimer that induces membrane curvature through tetramerization. Nature Communications 11, 5342 (2020).

10. Muhleip, A., McComas, S.E. \& Amunts, A. Structure of a mitochondrial ATP synthase with bound native cardiolipin. Elife 8, e51179 (2019).

11. Mühleip, A. et al. ATP synthase hexamer assemblies shape cristae of Toxoplasma mitochondria. Nature Communications 12, 120 (2021).

12. Gahura, O. et al. The $\mathrm{F}_{1}$-ATPase from Trypanosoma brucei is elaborated by three copies of an additional p18-subunit. FEBS J 285, 614-628 (2018).

13. Montgomery, M.G., Gahura, O., Leslie, A.G.W., Zikova, A. \& Walker, J.E. ATP synthase from Trypanosoma brucei has an elaborated canonical $\mathrm{F}_{1}$-domain and conventional catalytic sites. Proc Natl Acad Sci U S A 115, 2102-2107 (2018).

14. Serricchio, M. et al. Depletion of cardiolipin induces major changes in energy metabolism in Trypanosoma brucei bloodstream forms. FASEB J 35, 21176 (2020).

15. Muhleip, A.W., Dewar, C.E., Schnaufer, A., Kuhlbrandt, W. \& Davies, K.M. In situ structure of trypanosomal ATP synthase dimer reveals a unique arrangement of catalytic subunits. Proc Natl Acad Sci U S A 114, 992-997 (2017).

16. Schnaufer, A., Clark-Walker, G.D., Steinberg, A.G. \& Stuart, K. The F1-ATP synthase complex in bloodstream stage trypanosomes has an unusual and essential function. EMBO J 24, 4029-40 (2005).

17. Brown, S.V., Hosking, P., Li, J. \& Williams, N. ATP synthase is responsible for maintaining mitochondrial membrane potential in bloodstream form Trypanosoma brucei. Eukaryot Cell 5, 45-53 (2006).

18. Gahura, O., Hierro-Yap, C. \& Zikova, A. Redesigned and reversed: Architectural and functional oddities of the trypanosomal ATP synthase. Parasitology 148, 1151-1160 (2021).

19. Hierro-Yap, $C$. et al. Bioenergetic consequences of $\mathrm{F}_{0} \mathrm{~F}_{1}$-ATP synthase/ATPase deficiency in two life cycle stages of Trypanosoma brucei. J Biol Chem 296, 100357 (2021). 
20. Gahura, O., Panicucci, B., Vachova, H., Walker, J.E. \& Zikova, A. Inhibition of $\mathrm{F}_{1-}$ ATPase from Trypanosoma brucei by its regulatory protein inhibitor TbIF1. FEBS J 285, 4413-4423 (2018).

21. Zikova, A., Schnaufer, A., Dalley, R.A., Panigrahi, A.K. \& Stuart, K.D. The F(0)F(1)ATP synthase complex contains novel subunits and is essential for procyclic Trypanosoma brucei. PLoS Pathog 5, e1000436 (2009).

22. Perez, E. et al. The mitochondrial respiratory chain of the secondary green alga Euglena gracilis shares many additional subunits with parasitic Trypanosomatidae. Mitochondrion 19 Pt B, 338-49 (2014).

23. Sathish Yadav, K.N. et al. Atypical composition and structure of the mitochondrial dimeric ATP synthase from Euglena gracilis. Biochim Biophys Acta 1858, 267-275 (2017).

24. Dewar, C.E., Oeljeklaus, S., Warscheid, B. and Schneider, A. Characterisation of a highly diverged mitochondrial ATP synthase peripheral stalk subunit b in Trypanosoma brucei (2021).

https://www.biorxiv.org/content/10.1101/2021.10.13.464200v1

25. Aphasizheva, I. et al. Lexis and Grammar of Mitochondrial RNA Processing in Trypanosomes. Trends Parasitol 36, 337-355 (2020).

26. Blum, B., Bakalara, N. \& Simpson, L. A model for RNA editing in kinetoplastid mitochondria: "guide" RNA molecules transcribed from maxicircle DNA provide the edited information. Cell 60, 189-98 (1990).

27. Adler, B.K., Harris, M.E., Bertrand, K.I. \& Hajduk, S.L. Modification of Trypanosoma brucei mitochondrial rRNA by posttranscriptional 3' polyuridine tail formation. $\mathrm{Mol}$ Cell Biol 11, 5878-84 (1991).

28. Hofer, A., Steverding, D., Chabes, A., Brun, R. \& Thelander, L. Trypanosoma brucei CTP synthetase: a target for the treatment of African sleeping sickness. Proc Natl Acad Sci U S A 98, 6412-6 (2001).

29. Gupta, K. et al. The role of interfacial lipids in stabilizing membrane protein oligomers. Nature 541, 421-424 (2017).

30. Arnold, I., Pfeiffer, K., Neupert, W., Stuart, R.A. \& Schagger, H. Yeast mitochondrial $\mathrm{F}_{1} \mathrm{~F}_{\mathrm{o}}$-ATP synthase exists as a dimer: identification of three dimer-specific subunits. EMBO J 17, 7170-8 (1998).

31. Gu, J. et al. Cryo-EM structure of the mammalian ATP synthase tetramer bound with inhibitory protein IF1. Science 364, 1068-1075 (2019).

32. Spikes, T.E., Montgomery, M.G. \& Walker, J.E. Interface mobility between monomers in dimeric bovine ATP synthase participates in the ultrastructure of inner mitochondrial membranes. Proc Natl Acad Sci U S A 118, e2021012118 (2021).

33. Cadena, L.R. et al. Mitochondrial contact site and cristae organization system and $\mathrm{F}_{1} \mathrm{~F}_{\mathrm{o}^{-}}$ ATP synthase crosstalk is a fundamental property of mitochondrial cristae. mSphere $\mathbf{6}$, e0032721 (2021).

34. Davies, K.M. et al. Macromolecular organization of ATP synthase and complex I in whole mitochondria. Proc Natl Acad Sci U S A 108, 14121-6 (2011). 
35. Blum, T.B., Hahn, A., Meier, T., Davies, K.M. \& Kühlbrandt, W. Dimers of mitochondrial ATP synthase induce membrane curvature and self-assemble into rows. Proc Natl Acad Sci U S A 116, 4250-4255 (2019).

36. Bochud-Allemann, N. \& Schneider, A. Mitochondrial substrate level phosphorylation is essential for growth of procyclic Trypanosoma brucei. J Biol Chem 277, 32849-54 (2002).

37. Poon, S.K., Peacock, L., Gibson, W., Gull, K. \& Kelly, S. A modular and optimized single marker system for generating Trypanosoma brucei cell lines expressing T7 RNA polymerase and the tetracycline repressor. Open Biol 2, 110037 (2012).

38. Allemann, N. \& Schneider, A. ATP production in isolated mitochondria of procyclic Trypanosoma brucei. Mol Biochem Parasitol 111, 87-94 (2000).

39. Aibara, S., Dienemann, C., \& Cramer, P.. Structure of an inactive RNA polymerase II dimer. Nucleic Acids Research, gkab783 (2021).

40. de la Rosa-Trevin, J.M. et al. Scipion: A software framework toward integration, reproducibility and validation in 3D electron microscopy. J Struct Biol 195, 93-9 (2016).

41. Zhang, K. Gctf: Real-time CTF determination and correction. J Struct Biol 193, 1-12 (2016).

42. Cowtan, K. The Buccaneer software for automated model building. 1. Tracing protein chains. Acta Crystallogr D Biol Crystallogr 62, 1002-11 (2006).

43. Waterhouse, A. et al. SWISS-MODEL: homology modelling of protein structures and complexes. Nucleic Acids Res 46, W296-W303 (2018).

44. Williams, C.J., Headd, J.J., Moriarty, N.W., Prisant, M.G., Videau, L.L., Deis, L.N., Verma, V., Keedy, D.A., Hintze, B.J., Chen, V.B. and Jain, S. MolProbity: More and better reference data for improved all-atom structure validation. Protein Science, 27, 293-315 (2018).

45. Barad, B.A., Echols, N., Wang, R.Y.R., Cheng, Y., DiMaio, F., Adams, P.D. and Fraser, J.S. EMRinger: side chain-directed model and map validation for 3D cryo-electron microscopy. Nature methods, 12, 943-946 (2015).

46. Goddard, T.D. et al. UCSF ChimeraX: Meeting modern challenges in visualization and analysis. Protein Sci 27, 14-25 (2018).

47. Ho, B.K. \& Gruswitz, F. HOLLOW: generating accurate representations of channel and interior surfaces in molecular structures. BMC Struct Biol 8, 49 (2008). 

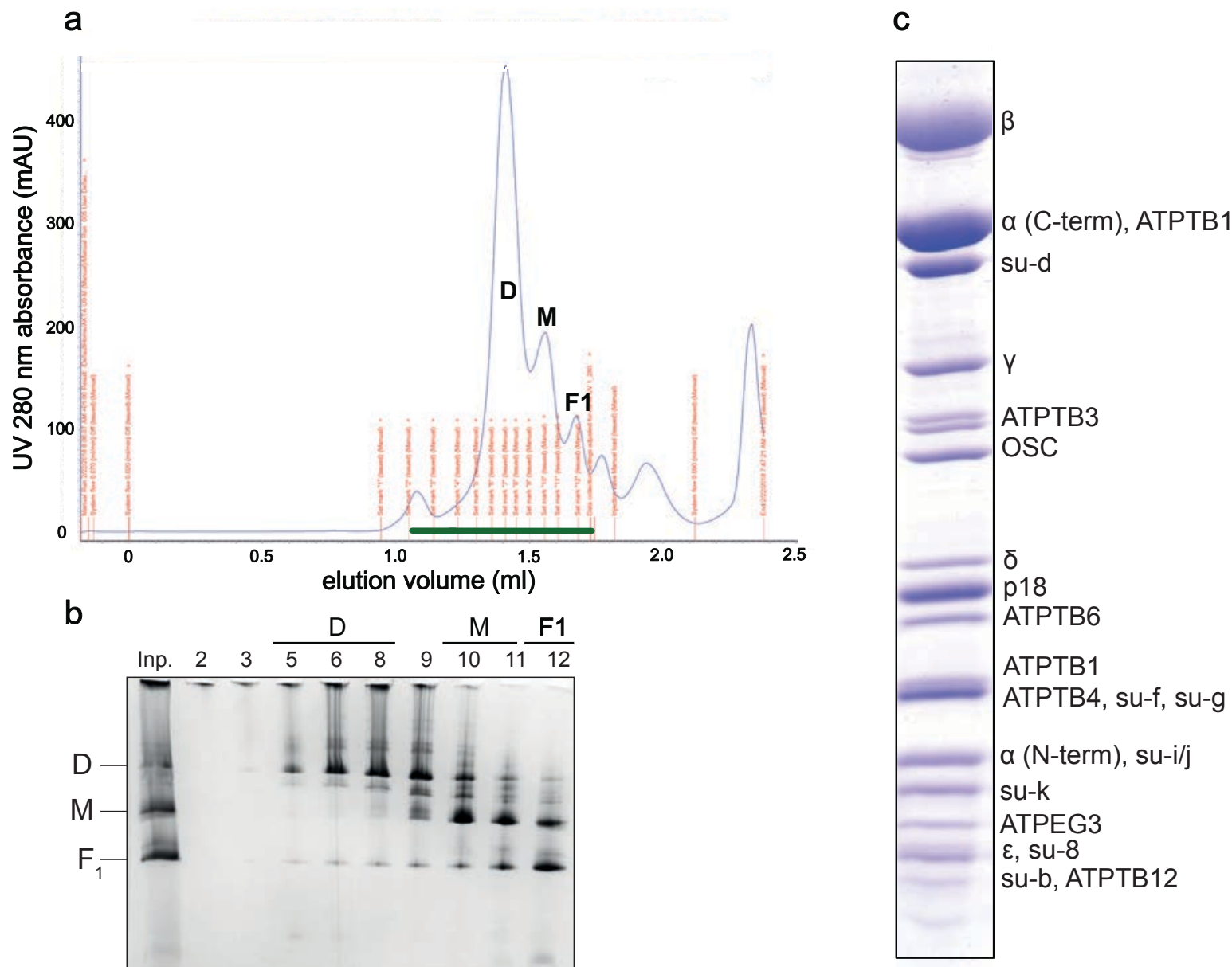

Extended Data Fig. 1 Purification of the T. brucei ATP synthase dimer.

a, Size exclusion chromatography trace with peaks enriched with ATP synthase dimers (D), monomers $(\mathrm{M})$ and $\mathrm{F}_{1}$-ATPase $\left(\mathrm{F}_{1}\right)$ labelled. b, Fractions from size exclusion chromatography marked with green bar in (a) resolved by native BN-PAGE. c, Dimer-enriched fraction resolved by SDS-PAGE stained by Coomassie blue dye. Bands are annotated based on mass spectrometry identification from excised gel pieces. 


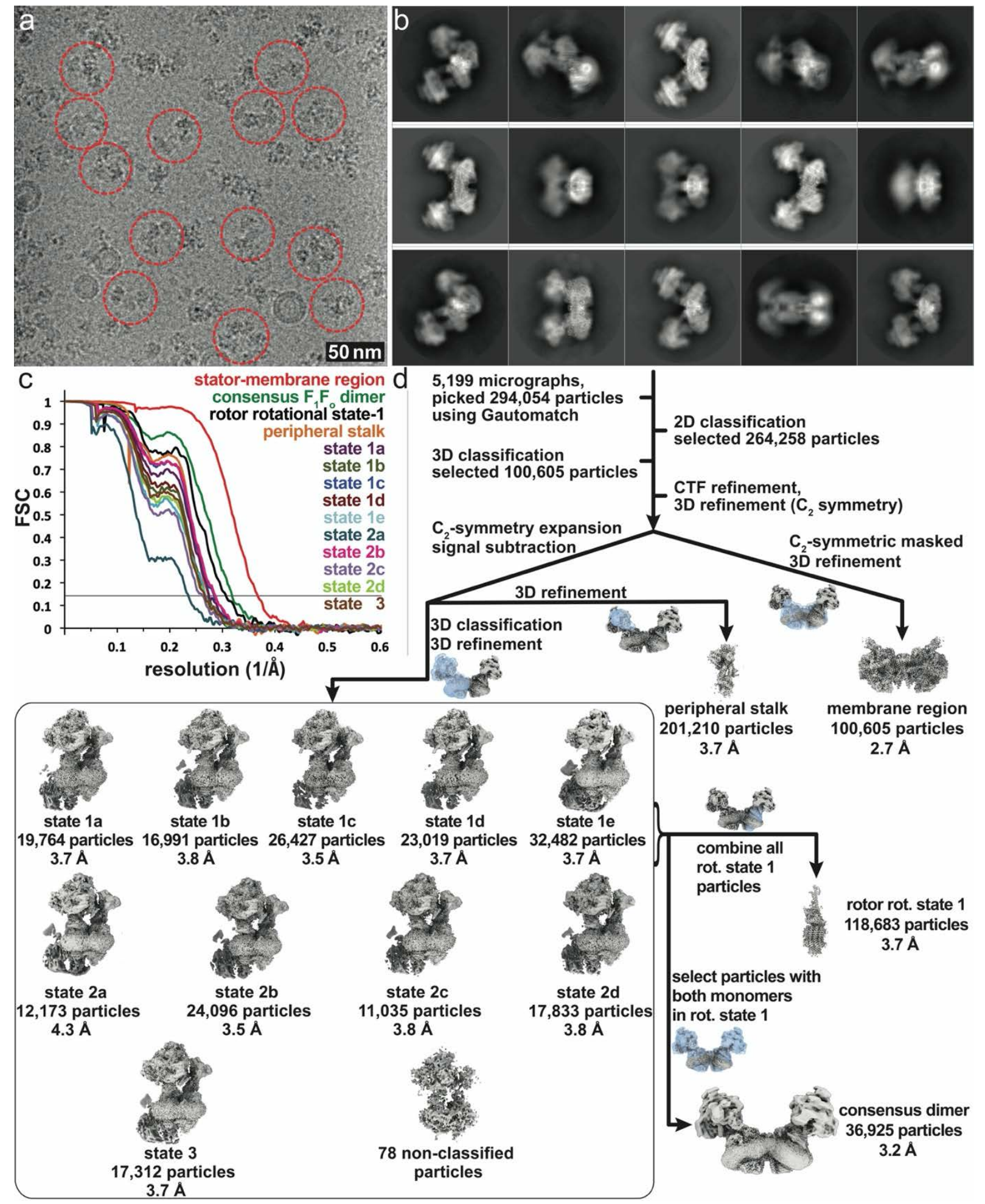

Extended Data Fig. 2 Cryo-EM data processing of the $T$. brucei ATP synthase dimer. a, Representative micrograph. b, 2D class averages. c, Fourier Shell Correlation (FSC) curves showing the estimated resolutions of ATP synthase maps according to the gold standard 0.143 criterion. d, Data processing scheme resulting in maps covering all regions of the complex, as well as 10 rotational states. 

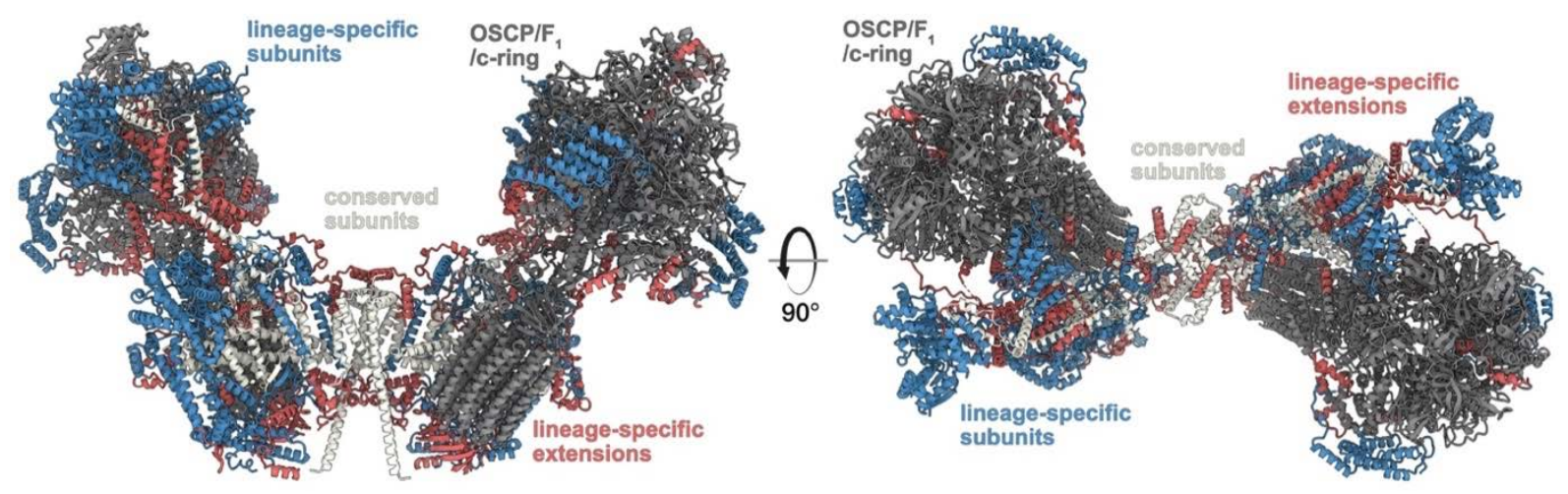

Extended Data Fig. 3 Conserved and phylum specific elements generate the T. brucei ATP synthase architecture.

The canonical $\mathrm{OSCP} / \mathrm{F}_{1} / c$-ring monomers (dark grey) are tied together by both conserved $\mathrm{F}_{\mathrm{o}}$ subunits and extensions of lineage-specific subunits (red). The $\mathrm{F}_{\mathrm{o}}$ periphery and peripheral stalk attachment are composed of lineage specific subunits (blue). 

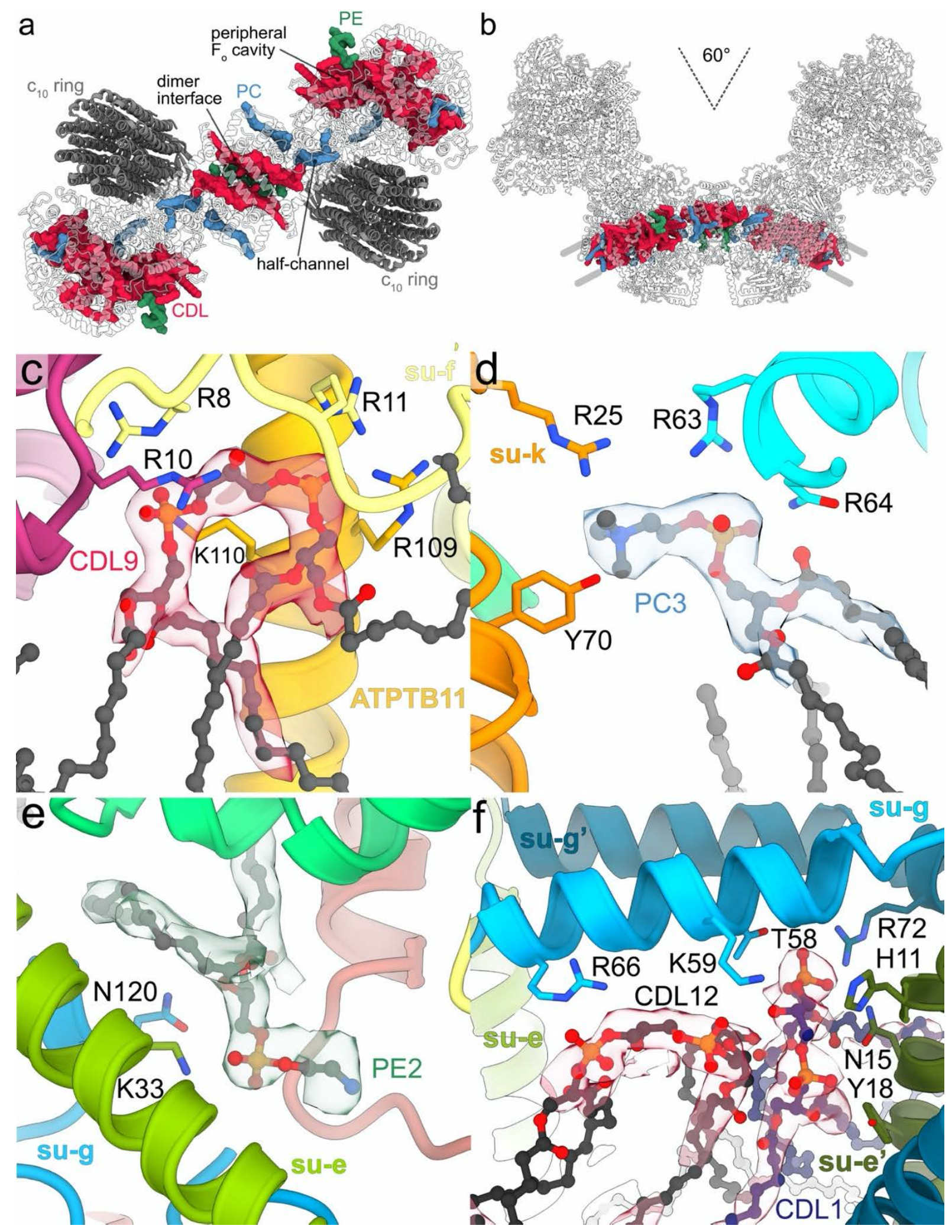

Extended Data Fig. 4 The $F_{0}$ region coordinates numerous bound lipids.

a, $F_{o}$ top view, cardiolipin (CDL), phosphatidylcholine (PC) and phosphatidlyethanolamine (PE) are bound at the dimer interface, the lumenal proton half-channel and the peripheral $\mathrm{F}_{\mathrm{o}}$ cavity. $\mathbf{b}$, The $60^{\circ}$-dimer angle generates a curved $\mathrm{F}_{\mathrm{o}}$ region with phospholipids bound in an arc-shaped bilayer. c-f, Bound lipids with cryo-EM density and coordinating residues. 

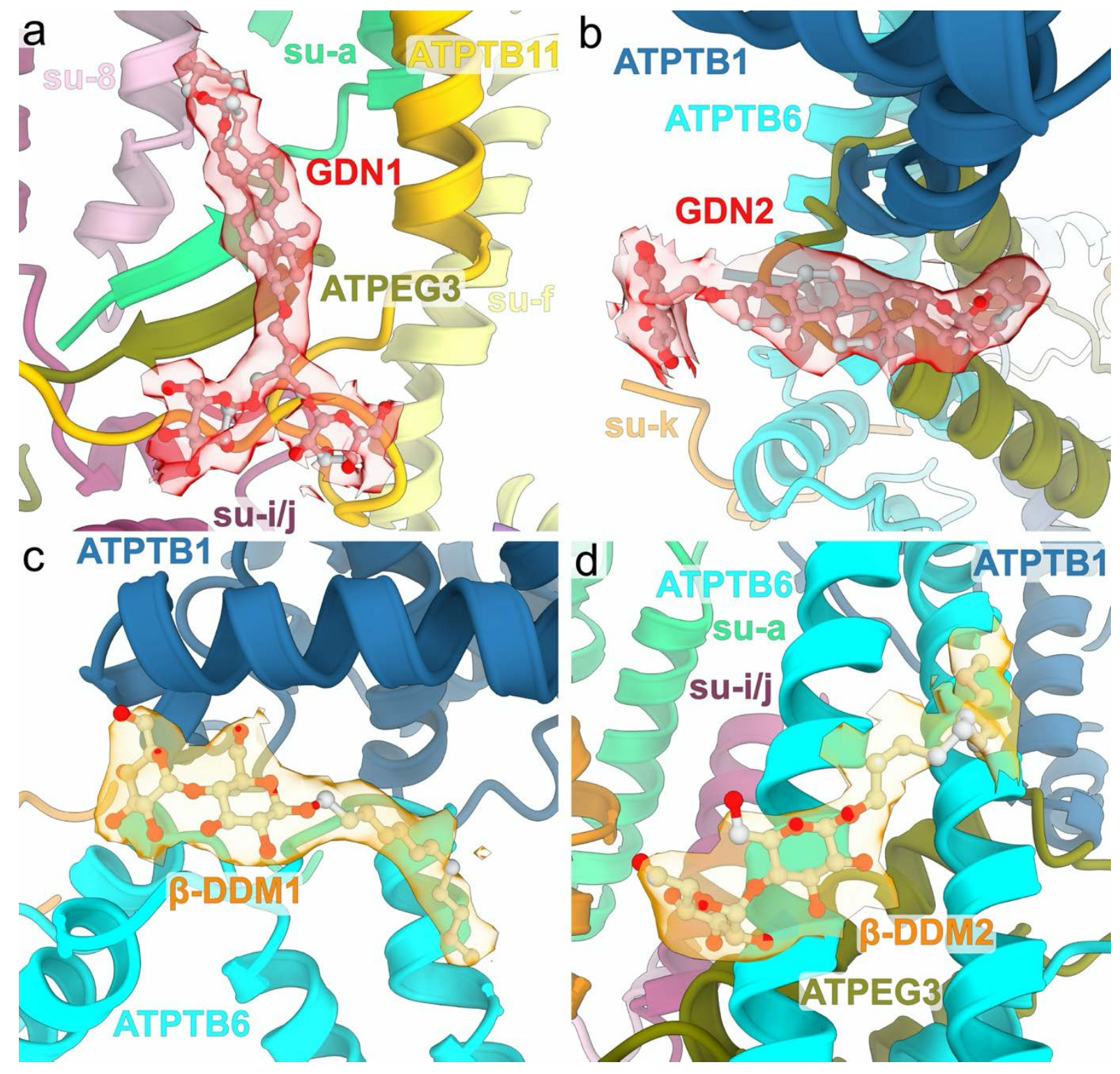

Extended Data Fig. 5 Bound detergents of the $F_{0}$ region.

GDN (a,b) and $\beta$-DDM (c,d) molecules bound in the periphery of the membrane region with cryo-EM map densities shown (transparent), indicating that both glycosides are retained in the detergent micelle. 


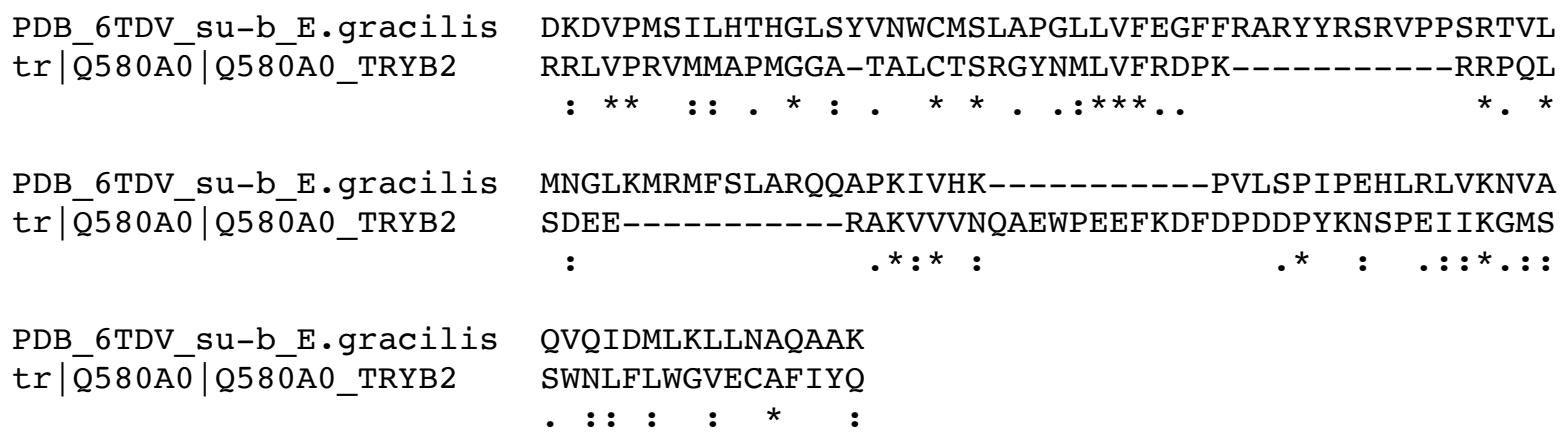

Extended Data Fig. 6 Sequence alignment of subunit-b with $E$. gracilis.

The $E$. gracilis sequence was retrieved from the PDB ID $6 \mathrm{TDV}^{1}$ and aligned with the current work using ClustalW ${ }^{2}$. Both sequences represent reduced versions of subunit- $b$ that are structurally conserved and occupy similar positions in the models. Termini were removed. 

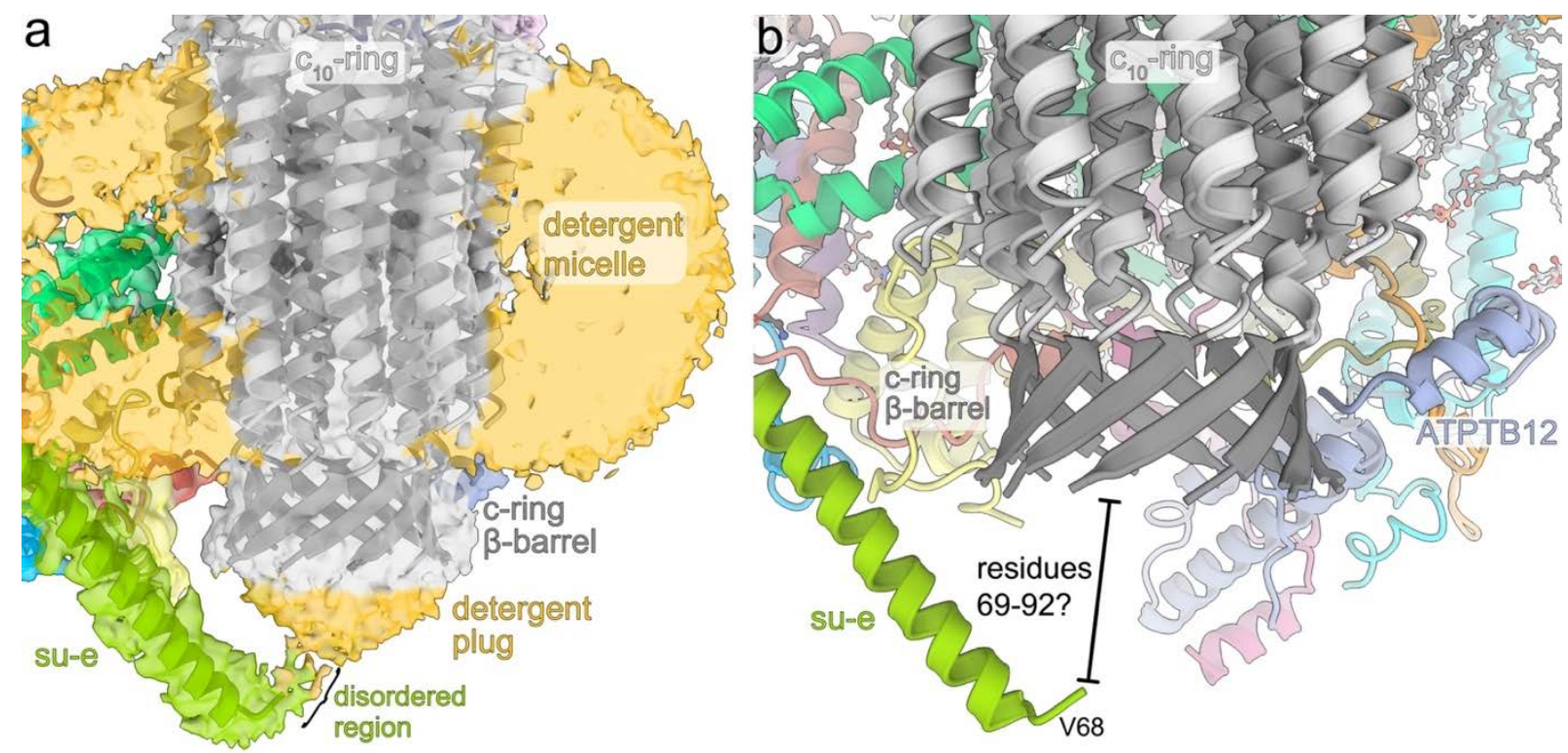

Extended Data Fig. 7 The C-terminal tail of subunit- $e$ interacts with the $c_{10}$-ring.

a, The cryo-EM map reveals disordered detergent density of the detergent belt surrounding the membrane region as well as a detergent plug on the luminal side of the $c$-ring. $\mathbf{b}$, The helical $\mathrm{C}$-terminus of subunit- $e$ extends into the lumen towards the $c$-ring. The terminal 23 residues are disordered and likely interact with the $\beta$-barrel. 


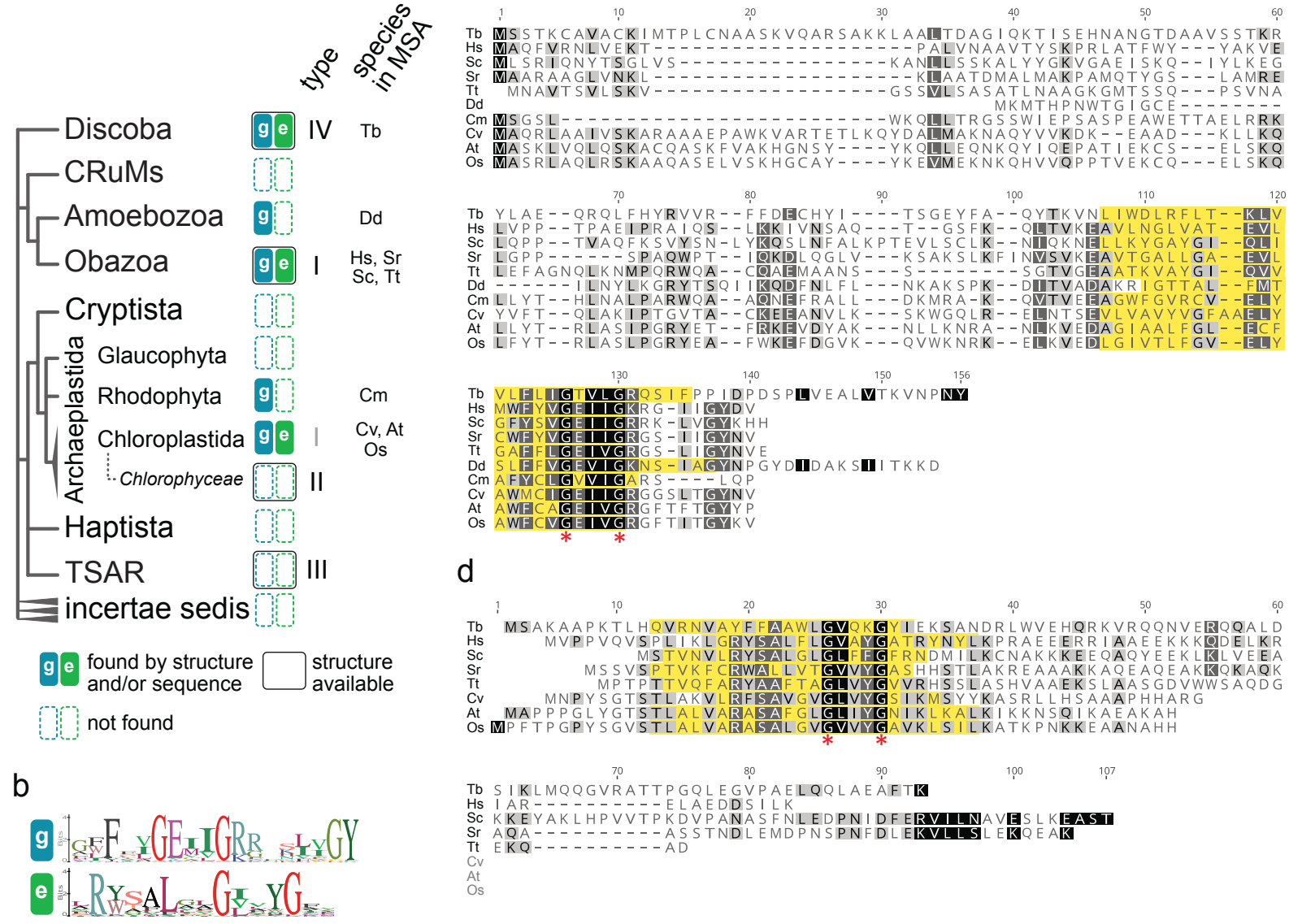

Extended Data Fig. 8 Phylogenetic distribution and sequence conservancy of subunit-e and $-g$.

a, Distribution of subunits $e$ and $g$ mapped on the phylogenetic tree of eukaryotes ${ }^{3}$. Homologs of subunits $e$ and $g$ were searched in non-redundant GenBank and UniprotKB protein databases by PSI-BLAST, and phmmer and $\mathrm{hmmsearch}^{4}$, respectively, using individual sequences of representatives from $H$. sapiens and $T$. brucei, and in the case of hmmsearch a multiple sequence alignment (MSA) of representatives from Homo sapiens, Saccharomyces cerevisiae, Arabidopsis thaliana and T. brucei, as queries. Groups, in which at least one structure of ATP synthase is available, are marked. Abbreviations of species used in MSA in panels (c) and (d) are shown. b, Sequence logo of GXXXG motifs and flanking regions of subunits $e$ and $g$. Hits from hmmsearch were clustered by CD-HIT Suite ${ }^{5}$ to $50 \%$ sequence identity and MSA of representative sequences of each cluster was generated by Clustal Omega $4^{6}$. The sequence logos were created from MSA in Geneious Prime (Biomatters Ltd.). c,d, MSA of sequences of subunits g (c) and e (d) from species representing major groups shown in (a) generated by MUSCLE $^{7}$ and visualized in Geneious Prime. The experimentally determined or predicted transmembrane regions are highlighted in yellow. Species abbreviations: $\mathrm{Tb}-T$. brucei, $\mathrm{Hs}-$ H. sapiens, $\mathrm{Sc}-S$. cerevisiae, $\mathrm{Sr}$ - Salpingoeca rosetta, $\mathrm{Tt}$ - Thecamonas trahens, Dd Dictyostelium discoideum, $\mathrm{Cm}$ - Cyanidioschyzon merolae, $\mathrm{Cv}-$ Chlorella vulgaris, At Arabidopsis thaliana, Os - Oryza sativa. 
a

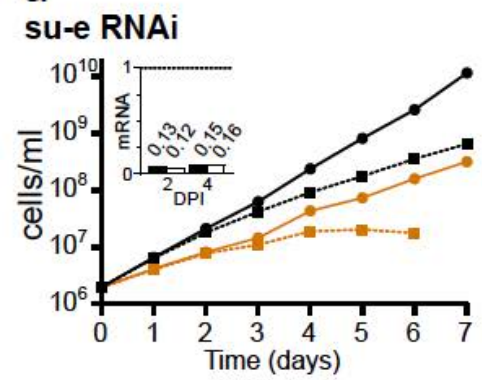

b

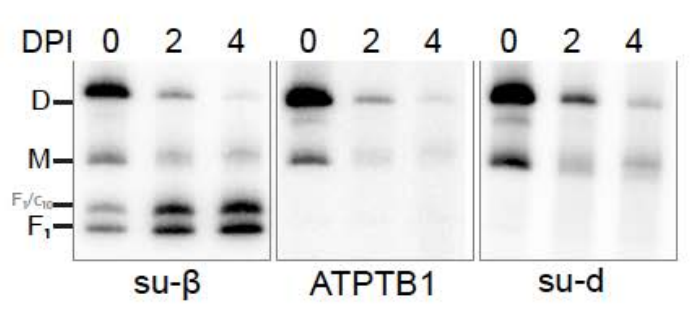

su-f RNAi

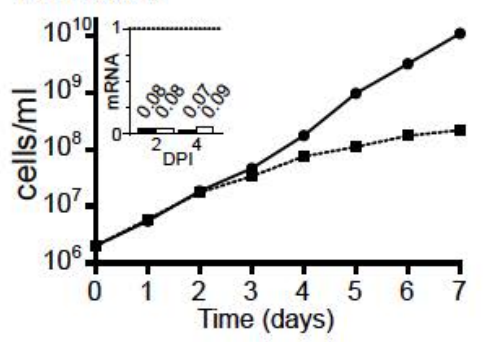

su-i/j RNAi

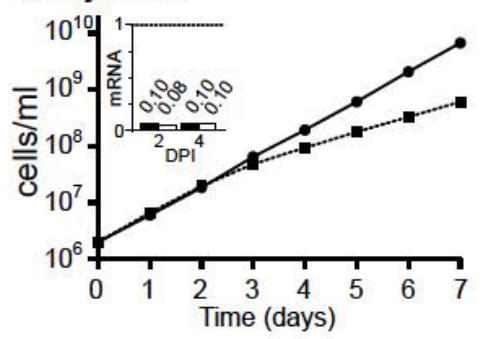

su-k RNAi

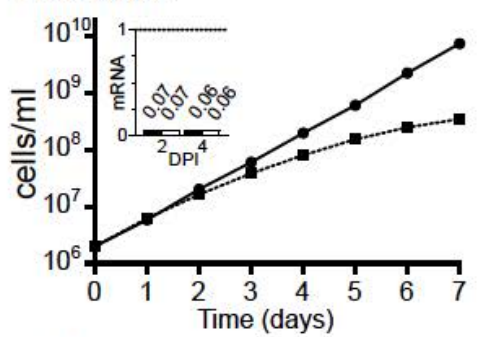

ATPTB3 RNAi

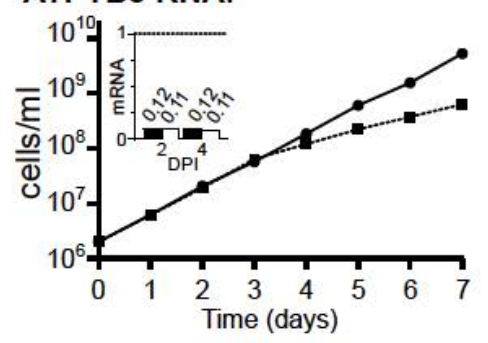

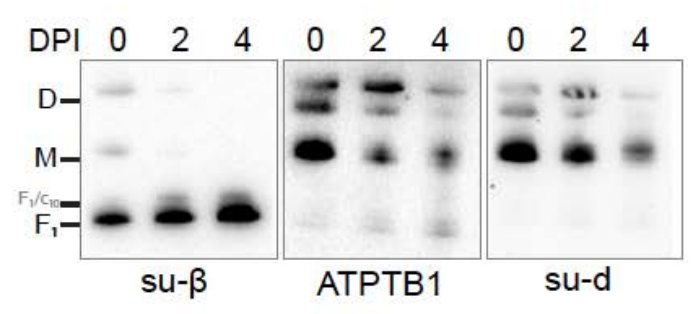
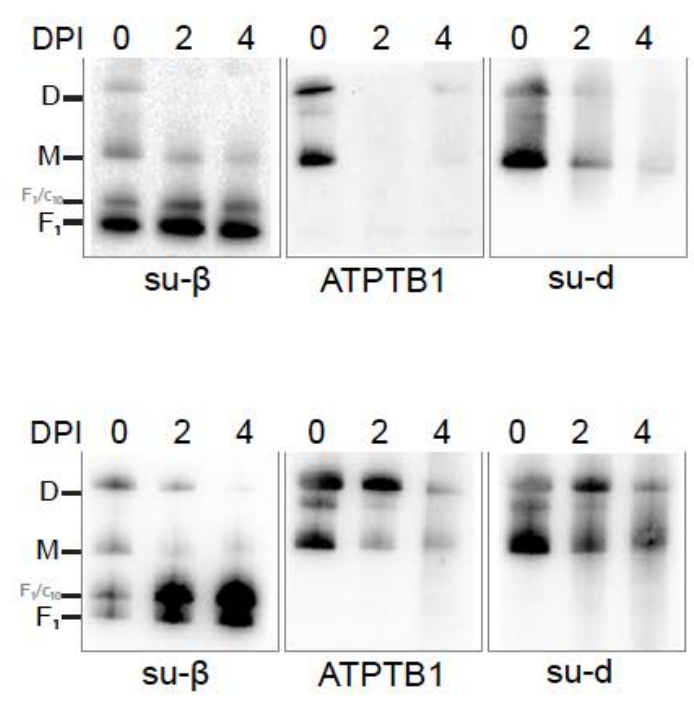

C
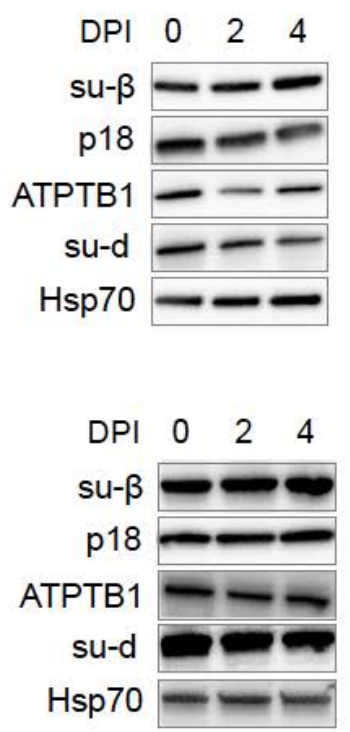

$\begin{aligned} \text { DPI } & 02 \\ \text { su- } \beta & - \\ \text { p18 } & - \\ \text { ATPTB1 } & - \\ \text { su-d } & - \\ \text { Hsp70 } & -\end{aligned}$
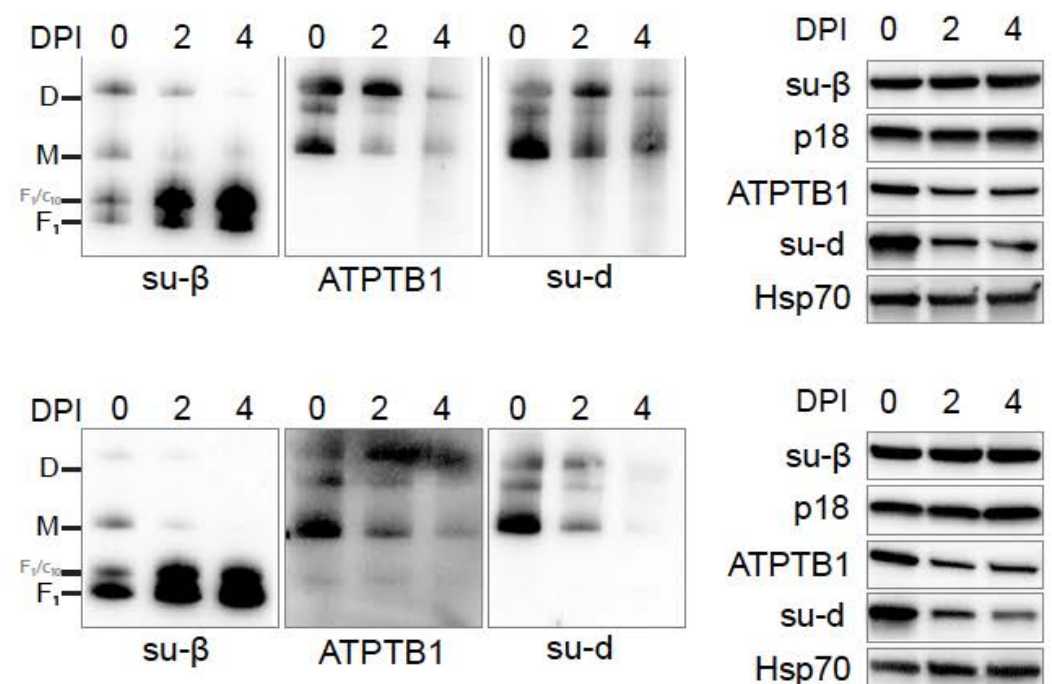

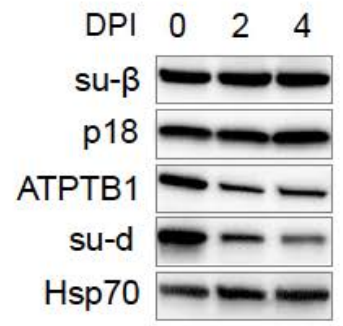


a

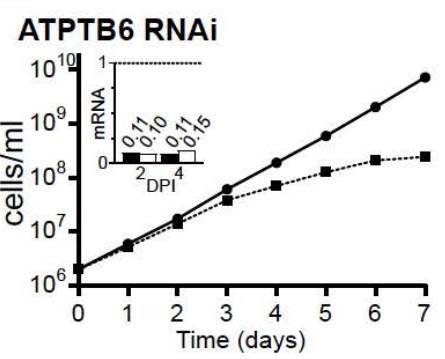

ATPTB12 RNAi

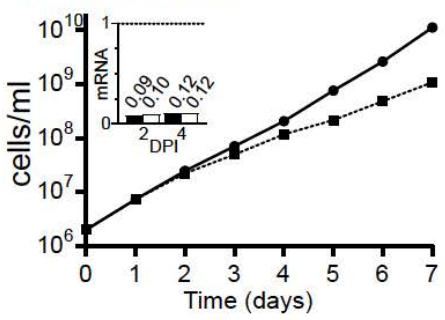

ATPTB14 RNAi

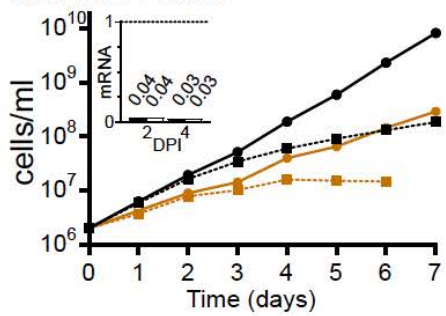

ATPEG3 RNAi

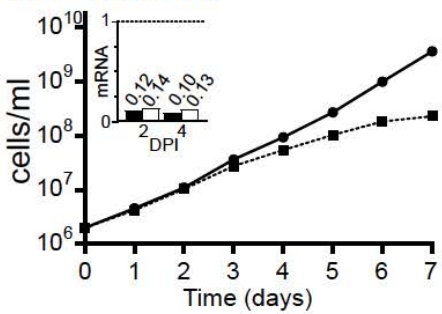

ATPEG4 RNAi

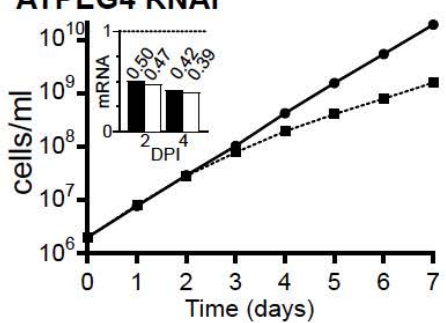

b
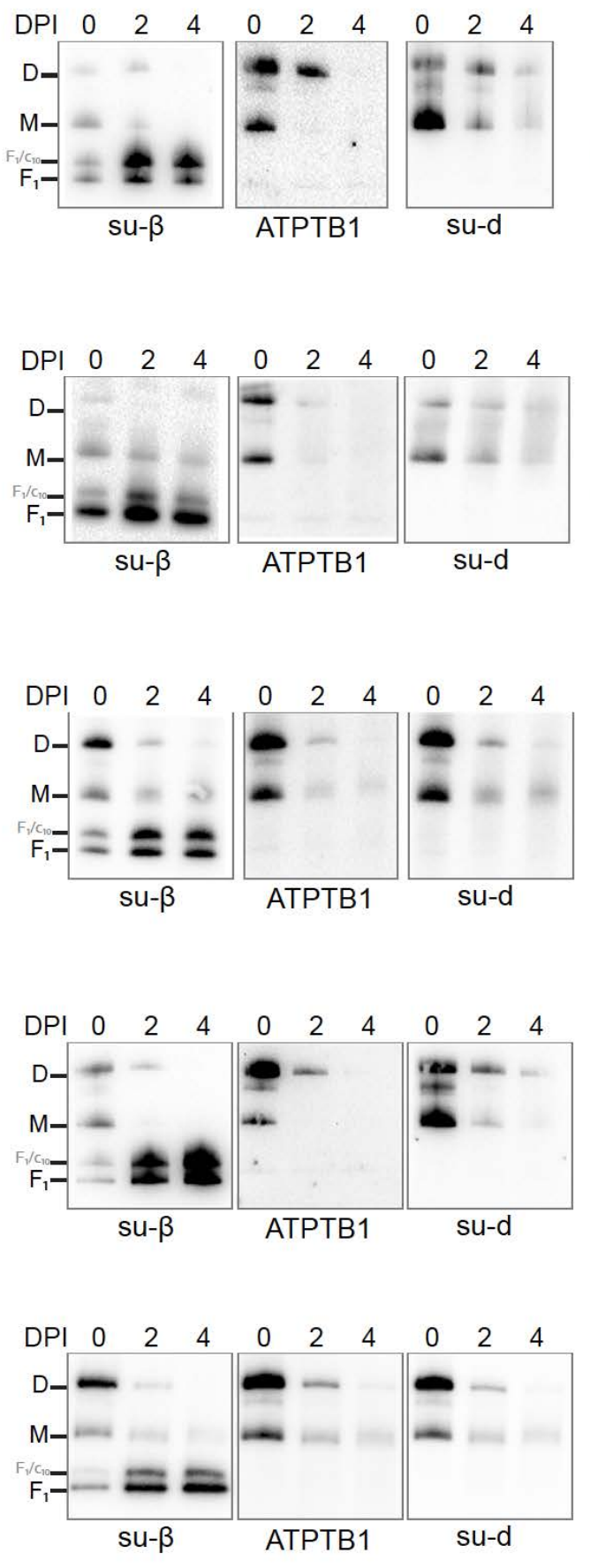

C
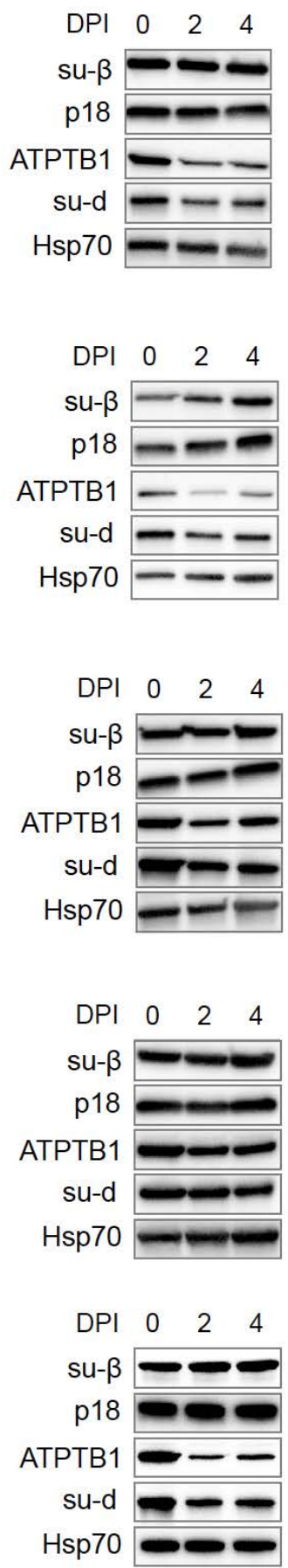

Extended Data Fig. 9 Effects of RNAi knock-down of ATP synthase subunits on viability and stability and dimerization of ATP synthase.

a, Growth curves of indicated non-induced (solid lines) and tetracycline induced (dashed lines) RNAi cells lines in the presence (black) or absence (brown) of glucose. The insets show relative levels of the respective target mRNA at indicated days post induction (DPI) normalized to the levels of 18S rRNA (black bars) or B-tubulin (white bars). b. Immunoblots of mitochondrial lysates from indicated RNAi cell lines resolved by BN-PAGE probed by antibodies against indicated ATP synthase subunits. c, Immunoblots of whole cell lysates from indicated RNAi cell lines probed with indicated antibodies. 


\begin{tabular}{|c|c|c|c|c|c|c|c|c|c|c|c|c|c|c|}
\hline & $\begin{array}{l}\text { Mem- } \\
\text { brane } \\
\text { region }\end{array}$ & Rotor & $\begin{array}{l}\text { Periphe- } \\
\text { ral stalk }\end{array}$ & $\begin{array}{l}\mathrm{F}_{1} \mathrm{~F}_{0} \\
\text { dimer }\end{array}$ & $\begin{array}{l}\text { Rot. } \\
\text { la }\end{array}$ & $\begin{array}{l}\text { Rot. } \\
\text { lb }\end{array}$ & $\begin{array}{l}\text { Rot. } \\
\text { lc }\end{array}$ & $\begin{array}{l}\text { Rot. } \\
\text { ld }\end{array}$ & $\begin{array}{l}\text { Rot. } \\
\text { le }\end{array}$ & $\begin{array}{l}\text { Rot. } \\
2 a\end{array}$ & $\begin{array}{l}\text { Rot. } \\
2 \mathrm{~b}\end{array}$ & $\begin{array}{l}\text { Rot. } \\
2 \mathrm{c}\end{array}$ & $\begin{array}{l}\text { Rot. } \\
\text { 2d }\end{array}$ & $\begin{array}{l}\text { Rot. } \\
3\end{array}$ \\
\hline \multicolumn{15}{|l|}{ Data collection } \\
\hline Microscope & \multicolumn{14}{|c|}{ Titan Krios } \\
\hline Voltage $(\mathrm{kV})$ & \multicolumn{14}{|c|}{300} \\
\hline Camera & \multicolumn{14}{|c|}{ K2 Summit } \\
\hline Magnification & \multicolumn{14}{|c|}{$165 \mathrm{kx}$} \\
\hline Exposure $\left(\mathrm{e}^{-} / \AA^{2}\right)$ & \multicolumn{14}{|c|}{33} \\
\hline Defocus range $(\mu \mathrm{m})$ & \multicolumn{14}{|c|}{-1.6 to -3.2} \\
\hline Pixel size $(\AA)$ & \multicolumn{14}{|c|}{0.83} \\
\hline Movies collected & \multicolumn{14}{|c|}{5,199} \\
\hline Frames per movie & \multicolumn{14}{|c|}{20} \\
\hline \multicolumn{15}{|l|}{ Data processing } \\
\hline Initial particles & \multicolumn{14}{|c|}{$100,605\left(C_{2}\right.$ symmetry-expanded: 201,210$)$} \\
\hline Final no. particles & 100,605 & 118,683 & 201,210 & 36,925 & 19,764 & 26,427 & 23,019 & 16,991 & 34,482 & 12,173 & 24,096 & 11,035 & 17,833 & 17.312 \\
\hline Symmetry & $\mathrm{C}_{2}$ & $\mathrm{C}_{1}$ & $\mathrm{C}_{1}$ & $\mathrm{C}_{2}$ & $\mathrm{C}_{1}$ & $\mathrm{C}_{1}$ & $\mathrm{C}_{1}$ & $\mathrm{C}_{1}$ & $\mathrm{C}_{1}$ & $\mathrm{C}_{1}$ & $\mathrm{C}_{1}$ & $\mathrm{C}_{1}$ & $\mathrm{C}_{1}$ & $\mathrm{C}_{1}$ \\
\hline Map resolution $(\AA)$ & 2.7 & 3.7 & 3.7 & 3.2 & 3.7 & 3.5 & 3.7 & 3.8 & 3.7 & 4.3 & 3.5 & 3.8 & 3.8 & 3.7 \\
\hline Sharpening B factor & -46.2 & -74.4 & -92.5 & -49.8 & -61.8 & -61.1 & -57.6 & -45.6 & -58.0 & -73.8 & -54.5 & -65.2 & -54.9 & -61.7 \\
\hline \multicolumn{15}{|l|}{ EMD ID } \\
\hline \multicolumn{15}{|l|}{$\begin{array}{l}\text { Model refinement } \\
\text { statistics }\end{array}$} \\
\hline CC (map/model) & 0.86 & 0.83 & 0.82 & 0.71 & 0.79 & 0.79 & 0.82 & 0.79 & 0.69 & 0.71 & 0.81 & 0.77 & 0.77 & 0.79 \\
\hline $\begin{array}{l}\text { Resolution } \\
\text { (map/model) }\end{array}$ & 2.65 & 3.4 & 3.68 & 3.13 & 3.48 & 3.56 & 3.36 & 3.55 & 3.57 & 3.94 & 3.39 & 3.73 & 3.64 & 3.58 \\
\hline No. of atoms & 76,690 & 19,669 & 12,083 & 251,552 & 129,568 & 129,568 & 129,568 & 129,568 & 129,568 & 129,563 & 129,563 & 129,563 & 129,563 & 129,566 \\
\hline No. of residues & 4074 & 1285 & 767 & 15,356 & 7872 & 7872 & 7872 & 7872 & 7872 & 7872 & 7872 & 7872 & 7872 & 7872 \\
\hline No. of lipids & 36 & 0 & 0 & 36 & 21 & 21 & 21 & 21 & 21 & 21 & 21 & 21 & 21 & 21 \\
\hline No. of ATP/ADP & 0 & 0 & 0 & 10 & 5 & 5 & 5 & 5 & 5 & 5 & 5 & 5 & 5 & 5 \\
\hline No. of Mg ions & 0 & 0 & 0 & 10 & 5 & 5 & 5 & 5 & 5 & 5 & 5 & 5 & 5 & 5 \\
\hline \multicolumn{15}{|l|}{ B-factor $\left(\AA^{2}\right)$} \\
\hline - protein & 54.05 & 56.13 & 77.88 & 84.48 & 55.65 & 70.37 & 80.22 & 83.27 & 70.70 & 112,72 & 79.93 & 65.52 & 66.49 & 101.5 \\
\hline - ligands & 50.57 & 58.25 & - & 69.94 & 40.99 & 72.29 & 63.18 & 78.43 & 63.76 & 75,25 & 74.47 & 61.79 & 46.55 & 83.68 \\
\hline Rotamer outliers (\%) & 0.44 & 0.40 & 0.31 & 0.22 & 0.42 & 0.09 & 0.18 & 0.26 & 0.58 & 0.18 & 0.27 & 0.48 & 0.42 & 0.39 \\
\hline \multicolumn{15}{|l|}{ Ramachandran (\%) } \\
\hline - outliers & 0.00 & 0.00 & 0.00 & 0.01 & 0.001 & 0.003 & 0.004 & 0.01 & 0.003 & 0.01 & 0.00 & 0.04 & 0.04 & 0.04 \\
\hline - allowed & 1.57 & 1.91 & 1.59 & 1.56 & 1.52 & 1.65 & 1.44 & 1.49 & 1.49 & 1.67 & 1.58 & 1.47 & 1.65 & 1.79 \\
\hline - favored & 98.43 & 98.08 & 98.41 & 98.42 & 98.47 & 98.34 & 98.56 & 98.49 & 98.48 & 98.31 & 98.42 & 98.49 & 98.31 & 98.17 \\
\hline Clash score & 1.66 & 2.44 & 2.32 & 2.26 & 2.60 & 2.65 & 2.53 & 2.67 & 2.99 & 2.38 & 2.30 & 2.52 & 2.38 & 3.57 \\
\hline MolProbity score & 0.92 & 1.03 & 1.01 & 1.00 & 1.05 & 1.05 & 1.04 & 1.05 & 1.09 & 1.02 & 1.01 & 1.04 & 1.02 & 1.15 \\
\hline \multicolumn{15}{|l|}{ RMSD } \\
\hline - bonds $(\AA)$ & 0.004 & 0.004 & 0.02 & 0.003 & 0.003 & 0.003 & 0.004 & 0.003 & 0.003 & 0.002 & 0.003 & 0.003 & 0.003 & 0.003 \\
\hline - angles $\left({ }^{\circ}\right)$ & 0.455 & 0.416 & 0.386 & 0.407 & 0.414 & 0.424 & 0.417 & 0.407 & 0.412 & 0.410 & 0.416 & 0.419 & 0.428 & 0.421 \\
\hline EMRinger score & 5.11 & 3.96 & 1.61 & 2.56 & 3.24 & 2.95 & 3.32 & 2.85 & 3.32 & 1.35 & 2.89 & 2.32 & 2.49 & 2.8 \\
\hline PDB ID & & & & & & & & & & & & & & \\
\hline
\end{tabular}

Extended Data Table 1. Data collection, processing, model refinement and validation

statistics. 


\begin{tabular}{|c|c|c|c|c|c|}
\hline $\begin{array}{l}\text { Subunit } \\
\text { name }\end{array}$ & $\begin{array}{c}\text { TriTrypDB Lister } \\
\text { strain } 427 \text { ID }\end{array}$ & $\begin{array}{c}\text { TriTrypDB } \\
\text { TREU927 strain ID }\end{array}$ & $\begin{array}{c}\text { Uniprot } \\
\text { TREU927 strain } \\
\text { ID }\end{array}$ & Residues & $\begin{array}{c}\text { Residues } \\
\text { built }\end{array}$ \\
\hline \multicolumn{6}{|c|}{$F_{1}$ subcomplex } \\
\hline$\alpha$ & $\begin{array}{l}\text { Tb427_070081800 } \\
\text { Tb427_070081900 }\end{array}$ & $\begin{array}{l}\text { Tb927.7.7420 } \\
\text { Tb927.7.7430 }\end{array}$ & Q57TX9 & 584 & $\begin{array}{l}45-151 \\
161-584\end{array}$ \\
\hline$\beta$ & Tb427_030013500 & Tb927.3.1380 & Q57XX1 & 519 & $26-514$ \\
\hline$\gamma$ & Tb427_100005200 & Tb927.10.180 & B0Z0F6 & 305 & $2-301$ \\
\hline$\delta$ & Tb427_060054900 & Tb927.6.4990 & Q586H1 & 182 & $22-182$ \\
\hline$\varepsilon$ & Tb427_100054600 & Tb427.10.5050 & N/A & 75 & $11-75$ \\
\hline p18 & Tb427_050022900 & Tb927.5.1710 & Q57ZP0 & 188 & $23-188$ \\
\hline \multicolumn{6}{|c|}{ Fo subcomplex } \\
\hline OSCP & Tb427_100087100 & Tb927.10.8030 & Q38AG1 & 255 & $\begin{array}{l}18-202 \\
208-255\end{array}$ \\
\hline$a$ & mt encoded & mt encoded & N/A & 231 & $1-231$ \\
\hline$b$ & Tb427_040009100 & Tb927.4.720 & Q580A0 & 105 & $26-105$ \\
\hline$c$ & $\begin{array}{l}\text { Tb427_100018700 } \\
\text { Tb427_110057900 } \\
\text { Tb427_070019000 }\end{array}$ & $\begin{array}{c}\text { Tb927.10.1570 } \\
\text { Tb927.11.5280 } \\
\text { Tb927.7.1470 }\end{array}$ & $\begin{array}{l}\text { Q38C84 } \\
\text { Q385P0 } \\
\text { Q57WQ3 }\end{array}$ & 118 & $41-118$ \\
\hline$d$ & Tb427_050035800 & Tb927.5.2930 & Q57ZW9 & 370 & $\begin{array}{l}17-325 \\
332-354\end{array}$ \\
\hline$e$ & Tb427_110010200 & Tb927.11.600 & N/A & 92 & $1-383$ \\
\hline$f$ & Tb427_030016600 & Tb927.3.1690 & Q57ZE2 & 145 & $2-136$ \\
\hline$g$ & Tb427_020016900 & Tb927.2.3610 & Q586X8 & 144 & $16-144$ \\
\hline$i / j$ & Tb427_030029400 & Tb927.3.2880 & Q57ZM4 & 104 & $2-104$ \\
\hline $\boldsymbol{k}$ & Tb427_070011800 & Tb927.7.840 & Q57VT0 & 124 & $20-124$ \\
\hline 8 & Tb427_040037300 & Tb927.4.3450 & Q585K5 & 114 & $29-114$ \\
\hline ATBTB1 & Tb427_100008400 & Tb927.10.520 & Q38CI8 & 396 & $1-383$ \\
\hline ATPTB3 & Tb427_110067400 & Tb927.11.6250 & Q385E4 & 269 & $2-269$ \\
\hline ATPTB4 & Tb427_100105100 & Tb927.10.9830 & Q389Z3 & 157 & $21-157$ \\
\hline ATPTB6 & Tb427_110017200 & Tb927.11.1270 & Q387C5 & 169 & $2-169$ \\
\hline ATPTB11 & Tb427_030021500 & Tb927.3.2180 & Q582T1 & 156 & $18-156$ \\
\hline ATPTB12 & Tb427_050037400 & Tb927.5.3090 & Q57Z84 & 101 & $5-100$ \\
\hline ATPEG3 & Tb427_060009300 & Tb927.6.590 & Q583U4 & 98 & $14-98$ \\
\hline ATPEG4 & N/A & Tb927.11.2245 & N/A & 62 & $1-62$ \\
\hline
\end{tabular}

Extended Data Table 2. Composition of T. brucei ATP synthase dimer. 


\begin{tabular}{|c|c|}
\hline Subunit & Primer pair sequences \\
\hline \multicolumn{2}{|r|}{ Primers for amplification of RNAi cassettes } \\
\hline $\boldsymbol{b}$ & $\begin{array}{l}\text { TAATCTCGAGGGTACCGTTGAGTGAGGAGGAACGGG } \\
\text { GCAGTCTAGAGGATCCTATCCCTTCCACCCACCACT }\end{array}$ \\
\hline$e$ & $\begin{array}{l}\text { TAATCTCGAGGGTACCGGGAGTACAGAAGGGCTACA } \\
\text { TAGATCTAGAGGATCCCGTGCACACCATCAGCTG }\end{array}$ \\
\hline$f$ & $\begin{array}{c}\text { ATACTCGAGGGTACCGTGAGTACCGCCTTTACGC } \\
\text { GCGTCTAGAGGATCCAGCACTGATCACCAAACTGC }\end{array}$ \\
\hline$g$ & $\begin{array}{l}\text { ACTGCTCGAGGGTACCACGCGGGAATTCAAAAGACC } \\
\text { GCGGTCTAGAGGATCCCGTTGCGGTGCTTGTCATTA }\end{array}$ \\
\hline$i / j$ & $\begin{array}{l}\text { TAATCTCGAGGGTACCGAATATCCGATGCATGCCGC } \\
\text { GCCGTCTAGAGGATCCACTTCGCTCTACTGCATGCA }\end{array}$ \\
\hline $\boldsymbol{k}$ & $\begin{array}{l}\text { ATTACTCGAGCCCGGGCGATCAGTGCAGGGGATTTT } \\
\text { GCCGTCTAGAGGATCCTTTCCTCGAAAACGCACACA }\end{array}$ \\
\hline 8 & $\begin{array}{l}\text { ATGACTCGAGGGTACCGGGCTATGGTGTGGTATTATGC } \\
\text { GACGTCTAGAGGATCCGCAGAAAACTCCCAACGACA }\end{array}$ \\
\hline АТРTB3 & $\begin{array}{l}\text { ACTGCTCGAGGGTACCAAAGAGGAGGTGAGGTCTGC } \\
\text { GCAGTCTAGAGGATCCCCCTAGGGTTCTTCGAAGCA }\end{array}$ \\
\hline ATPTB4 & $\begin{array}{l}\text { CTGACTCGAGGGTACCTTCCTTTTCTGCTGCATCGG } \\
\text { GCAGTCTAGAGGATCCCTCCTCGGGCTTCCAATTTG }\end{array}$ \\
\hline АТРТВ6 & $\begin{array}{l}\text { ACTGCTCGAGGGTACCCAACATGGCAGTATCCGGTG } \\
\text { GCAGTCTAGAGGATCCTTATTAGTGGCGGTGGTGGT }\end{array}$ \\
\hline АТРTB11 & $\begin{array}{l}\text { ACTGCTCGAGGGTACCGCGCTCGTCTTCTCCATTTC } \\
\text { GCAGAAGCTTGGATCCAGGTTGGGGTGTTTAGGGAG }\end{array}$ \\
\hline АТРТВ12 & $\begin{array}{l}\text { TAATCTCGAGGGTACCGACGCCATCAAAGGAATGCC } \\
\text { GCCGTCTAGAGGATCCAGCAGCCAACAAACAGACAA }\end{array}$ \\
\hline ATPEG3 & $\begin{array}{l}\text { TACACTCGAGGGTACCAAACCTGAAGGCCCTCACAC } \\
\text { GCAGTCTAGAGGATCCCTCTTTCGTGCCGCCTGATA }\end{array}$ \\
\hline & mers for quantification of mRNA levels by qPCR \\
\hline $\boldsymbol{b}$ & $\begin{array}{l}\text { CCAAGAGTGATGATGGCCCC } \\
\text { CGTTTAGGGTCGCGGAAAAC }\end{array}$ \\
\hline$e$ & $\begin{array}{l}\text { CAAGCCTTGCACACACTTTATG } \\
\text { CCGCAAAGAAGTACGCCAC }\end{array}$ \\
\hline$f$ & $\begin{array}{l}\text { TTTTCTACATACCGCAGCAGT } \\
\text { TACCATTCCATGCGCGTTG }\end{array}$ \\
\hline$g$ & $\begin{array}{l}\text { GCAATTGTGTGAGCTGAACG } \\
\text { TACTGGCCGCATTGCATAAC }\end{array}$ \\
\hline$i / j$ & AGAGTAAAAGCGCGCCTACG \\
\hline
\end{tabular}




\begin{tabular}{|c|c|}
\hline \multirow{2}{*}{$\boldsymbol{k}$} & CAGTTGGAAAACCGGTAGCC \\
\hline $\mathbf{8}$ & $\begin{array}{c}\text { ACACAAAACACTTCCAGCAGA } \\
\text { CGCTATGACGGACAGGTGT }\end{array}$ \\
\hline ATPTB3 & $\begin{array}{c}\text { GCTACGGCGACTTGGTGC } \\
\text { CGTCACCGCGTATTTGTCA }\end{array}$ \\
\hline ATPTB4 & AACGTTTATATCAGCGGGCG \\
& CTGTTTTGGTCTGCACACGA \\
\hline \multirow{2}{*}{ ATPTB6 } & CCAAACTTTGAAGCAGCGGA \\
& ATTCCTTGGATCCGCACCTT \\
\hline ATPTB11 & $\begin{array}{c}\text { TCGGCATAGGAGAAGTAACGA } \\
\text { GATTCGGTTTGGAACTTGCG }\end{array}$ \\
\hline \multirow{2}{*}{ ATPTB12 } & CAACGGCCCCACATTCTC \\
\hline ATPEG3 & ACACCGCGGTCATTCATTG \\
& GCACTTCATTCTCCCGACTG \\
\hline
\end{tabular}

Extended Data Table 3. List of primers used in the study. 


\begin{tabular}{|c|c|c|c|c|}
\hline Target & Туре & Reference & Dilution SDS-PAGE & Dilution BN-PAGE \\
\hline \multicolumn{5}{|c|}{ Primary antibodies } \\
\hline subunit- $\beta$ & rabbit polyclonal & 1 & $1: 2000$ & $1: 2000$ \\
\hline p18 & rabbit polyclonal & 1 & $1: 1000$ & - \\
\hline ATPTB1 & rabbit polyclonal & 1 & $1: 1000$ & $1: 1000$ \\
\hline subunit-d & rabbit polyclonal & 1 & $1: 1000$ & $1: 500$ \\
\hline mtHsp70 & mouse monoclonal & 2 & $1: 5000$ & - \\
\hline \multicolumn{5}{|c|}{ Secondary antibodies } \\
\hline \multicolumn{2}{|c|}{ goat anti-rabbit IgG HRP conjugate } & BioRad 1721019 & $1: 2000$ & $1: 2000$ \\
\hline \multicolumn{2}{|c|}{ goat anti-mouse IgG HRP conjugate } & BioRad 1721011 & $1: 2000$ & $1: 2000$ \\
\hline
\end{tabular}

Extended Data Table 4. List of antibodies used in the study. 


\section{Extended Data references:}

1. Muhleip, A., McComas, S.E. \& Amunts, A. Structure of a mitochondrial ATP synthase with bound native cardiolipin. Elife 8, e51179 (2019).

2. Larkin, M.A. et al. (2007). Clustal W and Clustal X version 2.0. Bioinformatics, 23, 29472948 (2007).

3. Burki, F., Roger, A.J., Brown, M.W. \& Simpson, A.G.B. The New Tree of Eukaryotes. Trends Ecol Evol 35, 43-55 (2020).

4. Protein Sequence Similarity Search. Curr Protoc Bioinformatics 60, 3151-31523 (2017).

5. Huang, Y., Niu, B., Gao, Y., Fu, L. \& Li, W. CD-HIT Suite: a web server for clustering and comparing biological sequences. Bioinformatics 26, 680-2 (2010).

6. Sievers, F. et al. Fast, scalable generation of high-quality protein multiple sequence alignments using Clustal Omega. Mol Syst Biol 7, 539 (2011).

7. Edgar, R.C. MUSCLE: multiple sequence alignment with high accuracy and high throughput. Nucleic Acids Res 32, 1792-7 (2004). 


\section{Supplementary Files}

This is a list of supplementary files associated with this preprint. Click to download.

- Video1.mp4

- Video2.mp4

- Video3.mp4 